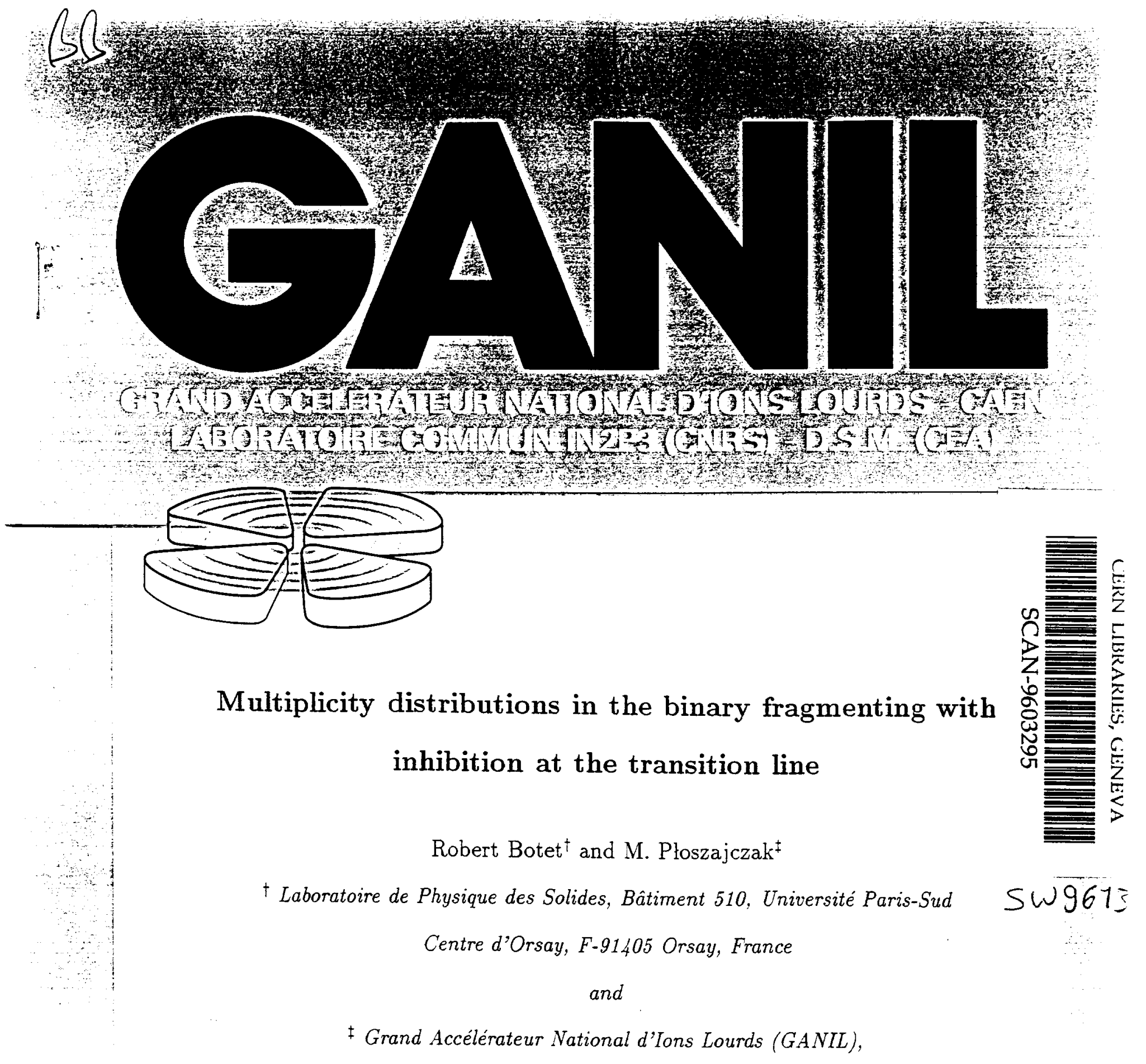
CEA/DSM - CNRS/IN2P3, BP 5027, F-14021 Caen Cedex, France

(March 9, 1996) 


\title{
Multiplicity distributions in the binary fragmenting with inhibition at the transition line
}

\author{
Robert Botet ${ }^{\dagger}$ and M. Ploszajczak ${ }^{\ddagger}$ \\ ${ }^{\dagger}$ Laboratoire de Physique des Solides, Bâtiment 510, Université Paris-Sud \\ Centre d'Orsay, F-91405 Orsay, France \\ and \\ ‡ Grand Accélérateur National d'Ions Lourds (GANIL), \\ CEA/DSM - CNRS/IN2P3, BP 5027, F-14021 Caen Cedex, France
}

(March 9, 1996)

\begin{abstract}
We investigate properties of the fragment multiplicity distribution obtained in the sequential binary fragmentation process at the transition line. We show that the multifragment cumulant correlation functions have the hierarchical, linked-pair structure. Several distinct classes of multiplicity domains are clearly identified and the asymptotic appearance of the Koba - Nielsen - Olesen scaling is discussed.

PACS numbers: $05.40 .+\mathrm{j}, 82.20 .-\mathrm{w}, 82.20 . \mathrm{Mj}$
\end{abstract}

Typeset using REVTEX 


\section{INTRODUCTION}

Most of the fragmenting systems are characterized by strongly off-equilibrium processes which cease due to dissipation. To take these features into account, the new kinetic fragmentation model has been proposed recently, where a dissipative process stops randomly the sequential fragmentation $[1,2]$. Sequential, conservative fragmentation is particularly interesting since it is believed to yield "universal" features, i.e. characteristic behaviours which do not depend on the precise mechanism governing the fragmentation. In the following, we shall consider the simplest version of this model called the Fragmentation - Inactivation Binary (FIB) model, where the kinetic rate-equation describe a purely binary process, i.e. any fragmenting cluster gives birth to exactly two fragments [3] .

Our aims in this work are limited to an understanding of the multiplicity distributions at the transition line between $\infty$-cluster and shattering phases of the FIB process $[1,2]$. (Multiplicity probability distributions in both $\infty$-cluster and shattering phases will be discussed in a separate paper [4]) . It is our experience that most of the gross measures of the cluster-mass (size) distribution do not discriminate among models unless supplemented with more fine grained information, especially correlations of various kinds. For that reason, we analyze in detail the supplementary information which could help to distinguish different fragmentation mechanisms and which are contained in the multiplicity distributions and

their scaling features. The generality of the fragmentation process as described by the FIB model permits to hope that the results of this paper should be relevant in different domains of physics : multihadron production in high energy collisions, nuclear multifragmentation process, polymer fragmentation, photoelectron count distribution in optics, the meteorite or asteroid fragmentation and, last but not least, the galaxy distribution. After all, the common feature of galaxy distributions, the quantum chromodynamic (QCD) evolution and the turbulent cascades, is an underlying scaling and branching mechanism, even if obscured in observable limits. All these systems are clearly dissipative and hence apt for a description by FIB process. 
After briefly discussing in chapter 2 some general features of the FIB model (for details see ref. [2]), we present in chapter 3 the cascade equations for the multiplicity evolution. Particular properties of these equations at the transition line of FIB model are discussed in details and the closed formulas for the higher order moments of the multiplicity distributions are given analytically. In chapter 4 , we discuss features of the multiplicity distributions which obey asymptotically the Koba - Nielsen - Olesen (KNO) scaling law. Detailed properties of the multiplicity distribution at the transition line are presented in chapter 5 . We show in particular the existence of several distinct domains of the multiplicity distributions : the Cayley domain $\left(0<p_{F}<1 / 2, \alpha>-1\right)$, the evaporative domain $\left(0<p_{F}<1, \alpha<-1\right)$, the Brand-Schenzle (BS) domain $\left(1 / 2<p_{F}<1, \alpha>-1\right)$ and discuss the appearance of the KNO asymptotic scaling. In chapter 6 we show that the asymptotic multiplicity distributions in the BS domain arise as a special solution of the stochastic equations of the multiplicative type. In chapter 7 , we investigate the structure of higher correlations in the FIB process at the transition line. We show in particular that the linked-pair ansatz for higher multifragment correlations holds exactly in all multiplicity domains and seems to be a basic property of a self-similar binary fragmentation process with inhibition. Finally, the main conclusions of the paper are given in chapter 8 .

\section{SOME FEATURES OF THE FIB MODEL}

In the FIB model, one deals with clusters characterized by some conservative scalar quantity, that shall be called the cluster mass. The anscestor cluster of mass $N$ is relaxing via an ordered and irreversible sequence of steps. The first step is either a binary fragmentation, say $(N) \rightarrow(j)+(N-j)$, occurring with the probability $p_{F}(N)$, or an inactivation $(N) \rightarrow(N)^{*}$ with probability $p_{I}(N)=1-p_{F}(N)$. Once inactive, the cluster cannot be reactivated anymore. The fragmentation leads to two fragments, with the mass partition probability $\sim F_{j, N-j}$. In the following steps, the relaxation process continues independently for each descendant cluster until either the low mass cutoff for monomers is reached or all 
clusters are inactive. Since for any event, the fragmentation and inactivation occur with the probabilities per unit of time $\sim F_{j, k-j}$ and $\sim I_{k}$ respectively, therefore the knowledge of the initial state and these two rate-functions $F$ and $I$, defines completely the fragmenting system and its evolution in the framework of the FIB model.

The composed particle first moment $\left.N_{C} \equiv 1-<n_{1}\right\rangle / N$, where $\left\langle n_{1}\right\rangle$ is the average number of monomers, plays the role of an order parameter when the total mass $N$ becomes infinite. If the probability $I_{N} / \Phi_{N}$ that no event occurs tends to 1 , then $N_{C} \rightarrow 1$. This is called the $\infty$ - cluster phase since there remains a large cluster of size of order $N$. On the contrary, when $I_{N} / \Phi_{N} \rightarrow 0$, then $N_{C}<1$, i.e. a finite ratio of the total mass is converted into finite-size clusters : monomers, dimers, etc. This is the shattered phase and the asymptotic $(N \rightarrow \infty)$ value of $N_{C}$ coincides with the total mass. Therefore, as $N \longrightarrow \infty$, one has in the FIB model a distinct second order phase transition associated with the shattering, i.e. the total destruction of an infinite cluster.

The second order shattering phase transition can be characterized by the following alternative reasoning. Each individual event is either a fragmentation or an inactivation. One can define the fragmentation probability $p_{F}$ attached to this choice without specifying the sizes of the descendants :

$$
p_{F}(k)=\sum_{i=1}^{k-1} F_{i, k-i}\left(I_{k}+\sum_{i=1}^{k-1} F_{i, k-i}\right)^{-1} .
$$

The inactivation probability is then : $p_{I}(k)=1-p_{F}(k)$. When the instability of the larger clusters is more important than the instability of the smaller ones, $p_{F}$ is an increasing function of the size and the system is in the shattered phase. Conversely, when the instability of the smaller clusters is more important, $p_{F}$ is a decreasing function of the fragment size and the system is in the $\infty$-cluster phase. The transition line is characterized by the rigorous independence of the probabilities $p_{F}$ and $p_{I}$ on the size of the considered object at any stage in the fragmentation avalanche. It should be underlined that the asymptotic $(t \rightarrow \infty)$ fragment mass distribution is independent of the functional form of the rate-functions $F$ and $I$ on the transition line, whereas it depends strongly on their form in both shattered 
and $\infty$-cluster phases.

The fragmentation process at the transition line of the shattering phase transition can be viewed as an externally driven process whereby the rate-functions $F_{j, k-j}$ and $I_{k}$ play the role of the driving noise. The fragmentation probability $p_{F}$ during the fragmentation avalanche has a fixed value between 0 and 1 , independent of time or the size of the fragmenting object [5]. This FIB process is a branching process, which can be mapped into the directed percolation on the Cayley tree (a mean-field percolation) [6]. Each node of a Cayley tree is occupied with a probability 1 and at each occupied point at time $l d t$ one chooses between three possibilities : fragmentation, inactivation, and 'no event' with respective probabilities $\tilde{p}_{0} \equiv p^{2}, \tilde{p}_{1} \equiv(1-p)^{2}$ and $\tilde{p}_{2} \equiv 2 p(1-p)$. At each fragmentation, a given ancestor cluster is replaced by two descendants and the fragmentation multiplicity increases by one unit. From one generation to the next, the number of fragments increases by a factor $\tilde{p}_{1}+2 \tilde{p}_{2}$ in the average. At criticality where the branching tree barely survives, $\tilde{p}_{0}=\tilde{p}_{2}=\left(1-\tilde{p}_{1}\right) / 2$, and therefore $p_{F}=1 / 2=p_{I}$. Let us note in passing that the FIB process at the transition line is analogous to the process of self-avoiding random walk, because the previously activated sites of this tree-like process 'repel' any subsequent reactivation [8] .

The mean-field limit in a broad class of dissipative coupled systems exhibiting the selforganized criticality (SOC) [10], can be described by such a critical branching process $[9,8]$. It was also demonstrated [11], that adding a simple perturbation mechanism to the relaxation rules of the FIB model, one obtains the FIB automaton which for any intial condition in the 'high-viscosity' region $\left(0<p_{F}<1 / 2\right)$ drives into the SOC state [10] without characteristic scales in space and time.

In the following, we shall assume the homogeneous multiplicative fragmentation kernel : $F_{i j} \propto(i j)^{\alpha}$, and the inactivation kernel $I_{k}$ in the form : $I_{k}=I_{1} k^{\beta}$. The transition line $\left(p_{F}(k)=\right.$ const $)$ in this case corresponds to : $\beta=2 \alpha+1$ if $\alpha>-2$ and $\beta=$ $\alpha-1$ if $\alpha<-2$. The size-distribution $\bar{n}_{s}(N)$ at the transition line is a power law : $\bar{n}_{s}(N) \sim N^{\tau-1} s^{-\tau} \quad(1 \ll k \ll N)$, and the exponent $\tau$ is always smaller than 2 . For $\alpha=0$ at the transition line $\tau=2 p_{F}$. The size-distribution in the whole shattered 
phase behaves also like a power law [1] : $\bar{n}_{s}(N) \sim N s^{\beta-2 \alpha-3} \quad(1 \ll k \ll N)$, with the exponent always larger than 2 independently of the strength $I_{1}$ of the inactivation rate [12]. The value of the exponent $\tau$, which is easiest to determine phenomenologically, does not fix unambiguously the parameters of fragmentation and inactivation kernels and hence does not correspond to a unique fragment multiplicity distribution. It is thus inevitable to carefully investigate the properties of the fragment multiplicity distribution in addition to the standard techniques of the fragment-size distribution in order to avoid a possible confusion, particularly dangerous in small and intermediate-size fragmenting systems such as atomic nuclei, fullerenes or metallic clusters.

\section{CASCADE EQUATION FOR THE MULTIPLICITY EVOLUTION}

The basic equations of the FIB process, relevant for the description of the fragmentation of an initial cluster (mass, energy etc.), such as master and cascade equations have been given before $[1,2]$. Here we discuss only those features of the FIB model which are relevant for the understanding of multiplicity distributions and their properties.

Let us define the multiplicity of an event at a time $t$ as the total number of fragmentations which occurred in this event until time $t$. Multiplicity defined in this way is then equal to the total number of fragments minus one, e.g. it equals 0 if no fragmentation occurred. Let us call $P_{N}[m ; t]$ the probability to get multiplicity $m$ at time $t$, starting from one cluster of size $N$ at time 0 . The time evolution equation for the multiplicity is given by the following integro-differential cascade equation :

$$
\begin{aligned}
P_{N}[m ; t] & =\delta(m) \exp \left(-\Phi_{N}(t)\right)+\delta(m)\left(1-p_{F}\right)\left(1-\exp \left(-\Phi_{N}(t)\right)+\right. \\
& +\int_{0}^{t} \exp \left(-\Phi_{N} t^{\prime}\right) d t^{\prime} \sum_{j=1}^{N-1} F_{j, N-j} \sum_{m^{\prime}=0}^{m-1} P_{j}\left[m^{\prime} ; t-t^{\prime}\right] P_{N-j}\left[m-m^{\prime}-1 ; t-t^{\prime}\right] .
\end{aligned}
$$

In the above equation, $\exp \left(-\Phi_{N} t\right)$ is the probability that no event occurs in the time interval $[0, t]$, and : 


$$
\Phi_{N} \equiv I_{N}+\sum_{i=1}^{N-1} F_{i, N-i}
$$

The parameter $p_{F}$ in (1) is generally a function of both $F_{i, j}$ and $\Phi_{N}$ rate functions and, with the exception of the transition line, $p_{F}$ depends explicitly on the cluster size. The meaning of the terms on the rhs of eq. (1) is the following. The first term is the probability that no fragmentation happens in the interval $[0, t]$. The second term describes the inactivation of the cluster $N$ in $[0, t]$. Finally, the third term corresponds to the fragmentation of the cluster $N$ into fragments $j$ and $N-j$ at time $t^{\prime}$ in the interval $[0, t]$. In terms of the generating function :

$$
G_{N}(x, t)=\sum_{m=0}^{\infty} P_{N}[m ; t] x^{m}=\sum_{k=0}^{\infty}<m^{k}>_{N} \frac{\ln ^{k} x}{k !}
$$

the evolution equation can be written in a compact form :

$$
\frac{1}{\Phi_{N}} \frac{\partial G_{N}}{\partial t}(x, t)+G_{N}(x, t)=\left(1-p_{F}\right)+\frac{x}{\Phi_{N}} \sum_{j=1}^{N-1} F_{j, N-j} G_{j}(x, t) G_{N-j}(x, t)
$$

with

$$
G_{1}(x, t)=1
$$

This equation provides a convenient way of deriving equations for the time-evolution of moments of the probability distribution $P_{N}[m]$. For example, for the factorial moments of $P_{N}[m]:$

$$
\tilde{F}_{k} \equiv F_{N}^{k}=<m(m-1) \ldots(m-k+1)>
$$

with the particular case $\tilde{F}_{0} \equiv F_{N}^{0}=1$, one gets the following evolution equations :

$$
\frac{1}{\Phi_{N}} \frac{\partial \tilde{F}_{k}}{\partial t}+\tilde{F}_{k}=\sum_{l=0}^{k}\left(\begin{array}{l}
k \\
l
\end{array}\right) \frac{1}{\Phi_{N}} \sum_{j=1}^{N-1} F_{j, N-j} F_{j}^{l}\left(F_{N-j}^{k-l}+(k-l) F_{N-j}^{k-l-1}\right)
$$

with

$$
F_{N}^{0}=1 \quad \text { and } \quad F_{1}^{k}=0
$$


Using the known relations between factorial moments and other families of moments such as the ordinary moments, central moments, cumulant moments or the factorial cumulant moments, one may obtain from (5) for each of this family the corresponding evolution equation. As each set of moments has its merits and most naturally describes certain distributions, there is not a preferred evolution equation and the choice depends on the example studied.

\section{KOBA - NIELSEN - OLESEN SCALING LAW}

In general, the form of the probability distribution $P_{N}[m]$ depends on the size of the fragmenting system $N$ and, hence, on the mean multiplicity of fragments $<m\rangle_{N}$. The behaviour of $P_{N}[m]$ for large $m$ and $\langle m\rangle_{N}$ is of interest for many reasons. In the context of strong interaction physics at very high energies, Koba, Nielsen and Olesen [14] suggested that the hadron multiplicity data for different average hadron multiplicities $<m>$ (different high energy collisions) should fall on the same curve when $<m>P_{m}\left(P_{m}\right.$ is the normalized probability to observe $m$ hadrons) is plotted against the scaled variable $m /<m\rangle$ :

$$
<m>P_{m}=f\left(\frac{m}{<m>}\right) \text {. }
$$

KNO prediction was based on assumption of validity of Feynman scaling for the many-body inclusive cross sections. Later, the relation of a KNO scaling to a phase transition in the Feynman-Wilson gas was emphasized [15] .

The KNO scaling limit is defined by the asymptotic behaviour of $<m>P_{m}$ as $m \rightarrow \infty$, $<m>\rightarrow \infty$ and $z \equiv m /<m>$ is fixed. The scaling function $f(z)$ must satisfy the normalization conditions :

$$
\begin{aligned}
& \int_{0}^{\infty} f(z) d z=1 \\
& \int_{0}^{\infty} z f(z) d z=1
\end{aligned}
$$

The latter condition fix : $\langle z\rangle=1$. Obviously, the moments of the scaling function : 


$$
<z^{i}>=\int_{0}^{\infty} z^{i} f(z) d z \equiv \frac{<m^{i}>}{<m>^{i}}
$$

are independent of the average multiplicity $\langle m\rangle$, and this is a characteristic feature of the KNO scaling. Besides $f(z)$, there is another scaling function $g(z) \equiv z f(z)$ which yields a different form of the KNO scaling law :

$$
m P_{m}=g\left(\frac{m}{<m>}\right)
$$

The scaling form (6) is satisfied by many distributions in the class of the Poisson transforms $[16]$ :

$$
P_{m}=\int_{0}^{\infty} d z f(z) \frac{(z<m>)^{m} \exp (-z<m>)}{m !}
$$

where :

$$
\begin{aligned}
\sum_{m=0}^{\infty} P_{m} & =1 \\
\sum_{m=0}^{\infty} m P_{m} & \equiv<m>
\end{aligned}
$$

If the weight function $f(z)$ in $(9)$ is suitably smooth then, independently of its specific form, the corresponding distribution $P_{m}$ satisfies the $\mathrm{KNO}$ scaling (6) for large $m$ and $<m>[17]$, i.e. an exact form of $P_{m}$ can be reconstructed from the asymptotic limit $f(z)$. The Bose-Einstein, negative binomial, Laguerre distributions are only few examples of physically important distributions in the class of Poisson transforms which satisfy the KNO scaling (6).

The scaling function $f(z)$ can itself be regarded as a probability distribution defined on the interval $0 \leq z<\infty$. It is then interesting to compare the moments of $P_{m}$ for various system sizes $<m>_{N}$, with those of $f(z)$ ( eq. (7)). For $P_{m}$ in the class of Poisson transforms (9), the ordinary moments $\left\langle z^{k}\right\rangle$ of $f(z)$ are related to the reduced factorial moments of $P_{m}$ :

$$
F_{k}=\frac{<m(m-1) \ldots(m-k+1)>}{<m>^{k}}=\left\langle z^{k}\right\rangle
$$


Similarly, the normalized cumulant moments of $f(z)$ are given by :

$$
\gamma_{p}^{(z)}=\int_{0}^{\infty}(z-<z>)^{p} f(z) d z=\frac{f_{p}}{\left(f_{1}\right)^{p}}
$$

where $f_{p}(p=1,2, \ldots)$ are the factorial cumulant moments of $P_{m}$ :

$$
\begin{aligned}
f_{1} & =<m>=\tilde{F}_{1} \\
f_{2} & =<m(m-1)>-<m>^{2}=\tilde{F}_{2}-\tilde{F}_{1}^{2} \\
f_{3} & =<m(m-1)(m-2)>-3<m(m-1)><m>+2<m>^{3} \\
& =\tilde{F}_{3}-3 \tilde{F}_{1} \tilde{F}_{2}+2 \tilde{F}_{1}^{3} \\
f_{4} & =<m(m-1)(m-2)(m-3)>-4<m(m-1)(m-2)><m>+ \\
& +12<m(m-1)><m>^{2}-3<m(m-1)>^{2}-6<m>^{4}+ \\
& =\tilde{F}_{4}-4 \tilde{F}_{3} \tilde{F}_{1}+12 \tilde{F}_{2} \tilde{F}_{1}^{2}-3 \tilde{F}_{2}^{2}-6 \tilde{F}_{1}^{4} \\
\ldots & =\ldots
\end{aligned}
$$

and $\tilde{F}_{k}$ are the factorial moments (4). Both $F_{p}$ and $f_{p}$ (or $\gamma_{p}^{(z)}$ ) moments will be shown to be averages over related correlation functions, with $f_{p}$ allowing the expression of the easily determined $F_{p}$ moments in terms of mostly lower order factorial cumulant moments. For distributions in the class of Poisson transforms and for large values of $\langle m\rangle, \gamma_{p}{ }^{(z)}$ should be constant independently of the precise value of $\langle m\rangle$.

Before further continuing discussion, one should examine meaning of the system size. From the point of the KNO multiplicity scaling law, the size of the system is given by the average fragment multiplicity $\langle m\rangle$. On the other hand, in the kinetic fragmentation models such as e.g. the FIB model, the natural size of the system is given by the initial mass $N$. The important question is then how does the fragment multiplicity $\langle m\rangle$ depends on $N$ for given fragmentation and inactivation rate functions, or equivalently for given the fragmentation probability $p_{F}$ and the asymmetry exponent $\alpha$. If $\langle m\rangle_{N}$ is a monotonous function of $N$, then there is one to one correspondence between $\langle m\rangle-$ and $N-$ size scales and a possibility of the multiplicity scaling law in the fragmentation process can be 
safely discussed. Otherwise, if $\left\langle m>_{N}=\operatorname{const}(N)\right.$, then changing of $<m>$ means changing of the rate functions (or $p_{F}$ and $\alpha$ ) of the fragmentation process and, hence, also the asymptotic multiplicity distribution. In this case, there is no reason whatsoever for an existence of a unique scaling function : $f\left(m /<m>_{N}\right)=<m>_{N}{ }^{\left(p_{F}, \alpha\right)} P_{m}\left(<m>_{N}\right)$ for different $<m>_{N}$. In other words, eventhough $\gamma_{p}$ is independent of $N$ for fixed $p_{F}$ and $\alpha$, the multiplicity distribution neither belongs to the class of Poisson transforms, nor it obeys the KNO scaling. In such a case, the normalized cumulant moments of $f(z)$ are not equal to the normalized factorial cumulant moments of the corresponding multiplicity distribution $P_{m}$.

An important feature holds when the generating function of the multiplicity probability distribution depends on a single complex variable $x^{<m>_{N}}$ :

$$
G_{N}(x, t)=\sum_{m=1}^{\infty} P_{N}[m ; t] x^{m}=F\left(x^{<m>N}\right) \quad,
$$

In this case, since this generating function is nothing but the Laplace transform of $P_{N}[m]$, the probability to get a multiplicity $m$ is just :

$$
<m>_{N} P_{N}[m]=\frac{1}{2 i \pi} \int_{c-i \infty}^{c+i \infty} F\left(e^{-u}\right) e^{u\left(m /<m>_{N}\right)} d u
$$

where $c$ is a complex constant having a positive real part. The condition expressed in (12) is then sufficient to get the KNO scaling, provided the integral (13) is defined. We shall return to this problem in the next section when discussing the scaling properties of the FIB process at the transition line. 


\section{MULTIPLICITY PROBABILITY DISTRIBUTIONS AT THE TRANSITION \\ LINE}

Let us study the generic case : $F_{i j}=(i j)^{\alpha}$, and focus on the asymptotic regime $t \rightarrow \infty$. As it will be shown below, various classes of the multiplicity distributions can be defined in different domains of the parameters $p_{F}$ and $\alpha$.

On the transition line, the asymptotic cluster size distribution is a power law with the exponent $\tau$ depending on values of both the fragmentation probability $p_{F}$ and the exponent $\alpha$ of the fragmentation kernel in a following way [2] :

$$
\frac{\Gamma(\tau+\alpha)}{\Gamma(\tau+2 \alpha+1)}=\frac{1}{p_{F}} \frac{\Gamma(\alpha+2)}{\Gamma(2 \alpha+3)}
$$

On the other hand, from (5) one obtains the recurrence relation for the multiplicity average :

$$
<m>_{N}=p_{F}+\frac{2}{\Phi_{N}} \sum_{j=1}^{N-1} j^{\alpha}(N-j)^{\alpha}<m>_{j}
$$

with

$$
<m>_{1}=0 .
$$

For the power law trial function in the case $\alpha>-1$ (the case $\alpha<-1$ will be treated in sect. 5.D), one finds :

$$
<m>_{N} \sim a N^{b}
$$

with two solutions :

$$
b=0 \longrightarrow<m>_{N} \sim \frac{p_{F}}{1-2 p_{F}}
$$

which is valid for $p_{F}<1 / 2$, and :

$$
b>0 \longrightarrow<m>_{N} \sim N^{b},
$$

which is valid for $p_{F}>1 / 2$. In the latter case, exponent $b$ can be obtained by solving the relation deduced from (15): 


$$
2 p_{F} \frac{\Gamma(\alpha+b+1)}{\Gamma(2 \alpha+b+2)} \frac{\Gamma(2 \alpha+2)}{\Gamma(\alpha+1)}=1
$$

By comparing with (14) we see that eq. (18) implies :

$$
b=\tau-1
$$

Hence for $p_{F}<1 / 2$ the fragment multiplicity is a constant independent of $N$, whereas for $p_{F}>1 / 2$ the fragment multiplicity is an algebraic function of $N$ :

$$
<m>_{N} \sim a N^{r-1}
$$

Finally, for $p_{F}=1 / 2$ one finds :

$$
<m>_{N} \sim \frac{1}{2} \ln N
$$

One can also obtain from (5) the generalization of the recurrence relation for higher order multiplicity moments. An example of that will be shown below in the case of the BS fragmentation regime.

\section{A. Brand-Schenzle fragmentation regime: $p_{F}>1 / 2, \alpha>-1$}

Let us generalize the above solutions for the average multiplicity $<m>_{N}$ (eq. (19)) to the case of higher order $(k>1)$ multiplicity moments. For that purpose, let us write :

$$
\tilde{F}_{k} \sim \frac{1}{p_{F}} \frac{k !\left[a p_{F} \Gamma(\alpha+\tau)\right]^{k}}{\Gamma(c(k)+\alpha+1)} N^{c(k)} \psi_{k}
$$

where $c(k)$ is an unknown function of both $k$ and $\psi_{k}$, and $a$ is the coefficient in eq. (19). Eq. (5) for $\tilde{F}_{k}$ in the limit $t \rightarrow \infty$, yields in this case :

$$
c(k)=c(l)+c(k-l)
$$

which, knowing that $c(1)=\tau-1$, leads to:

$$
c(k)=k(\tau-1)
$$


Moreover :

$$
\frac{\Gamma(k(\tau-1)+2 \alpha+2)}{\Gamma(k(\tau-1)+\alpha+1)} \frac{\Gamma(\alpha+1)}{\Gamma(2 \alpha+2)} \psi_{k}=\sum_{l=0}^{k} \psi_{l} \psi_{k-l}
$$

with

$$
\psi_{0}=p_{F} \quad \text { and } \quad \psi_{1}=1
$$

Equation (22) leads to :

$$
\psi_{k} \sim\left(\frac{\Gamma(\alpha+1)}{(\tau-1)^{\alpha+1}}\right)^{k-1} k^{\alpha}
$$

where $k \gg 1$. In particular, we can remark that all $\psi_{k}$ 's diverge when $\tau$ approaches 1 , i.e. when $p_{F} \rightarrow 1 / 2$. An important case is $\alpha=0$, for which the solution of $(22)$ is :

$$
\psi_{k}=(\tau-1)^{1-k} \quad(k \geq 1)
$$

Using (22) for any value of $\alpha$, one can calculate for example the asymptotic ordinary scaled moments of the multiplicity distribution :

$$
\frac{<m^{k}>_{N}}{<m>_{N}^{k}} \sim \frac{\sqrt{(\tau-1)}}{p_{F} \Gamma(\alpha+1)}\left(k^{2-\tau} \frac{p_{F} \Gamma(\alpha+\tau) \Gamma(\alpha+1)}{e^{2-\tau}(\tau-1)^{\alpha+\tau}}\right)^{k}
$$

where $N \gg 1$ and $k \gg 1$. Since this ratio is independent of the average multiplicity $<m>_{N}$, the generating function $G_{N}(x)$ is a function of the variable $x^{<m>_{N}}$ and according to (12) and (13) the KNO scaling holds. The relation (23) can be approximated around the maximum of the multiplicity probability distribution as follows :

$$
\frac{<m^{k}>_{N}}{<m>_{N}^{k}}=\frac{\Gamma\left(\frac{a+1+k}{c}\right)}{\Gamma\left(\frac{a+2}{c}\right) b^{k-1}},
$$

what immediately suggests the form of the scaling function :

$$
f(z)=A z^{a} \exp \left(-(b z)^{c}\right)
$$

in the BS domain. Comparing (24) with (23), one can determine the coefficients $a$ and $c$ of the KNO scaling function in the BS fragmentation regime which reads : 


$$
f(z)=A z^{\frac{1}{2(2-\tau)}-1} \exp \left(-(b z)^{\frac{1}{2-\tau}}\right)
$$

where $A$ is a normalization constant and :

$$
b=(2-\tau)^{2-\tau}(\tau-1)^{\tau-1}
$$

The most probable value $\hat{z}_{0}$ of the scaling function (26) as a function of $\tau$ is given by :

$$
\hat{z}_{0}=\left\{\begin{array}{l}
0 \quad \text { for } \quad \tau \leq 3 / 2 \\
(\tau-1)^{-(\tau-1)}[2(2-\tau)]^{-(2-\tau)}(2 \tau-3)^{2-\tau} \quad \text { for } \quad \tau>3 / 2
\end{array}\right.
$$

The behaviour of $\hat{z}_{0}$ at $\tau=3 / 2$ is reminiscent of an equilibrium phase transition if we interpret $\hat{z}_{0}$ as some kind of the order parameter. Together with (14), the relation (27) demonstrates also an implicit dependence of the most probable value $\hat{z}_{0}$ of the scaling function $f(z)$ both on the fragmentation probability $p_{F}$, i.e. on the strength of the external driving noise, and on the degree of asymmetry $\alpha$ of the fragmentation kernel through the dependence of $\tau$ on both $p_{F}$ and $\alpha$. Fig. 1 shows the diagram $p_{F}-\alpha$ with all fragmentation regions as well as the line $\tau=3 / 2$ :

$$
p_{F}=\frac{1}{2^{2 \alpha+3}} \frac{\Gamma^{2}(\alpha+1) \Gamma(4 \alpha+4)}{\Gamma^{3}(2 \alpha+2)},
$$

separating the two phases (27) in the BS fragmentation regime. Few typical multiplicity distributions for various values of $\tau$ are shown in Fig. 2. If not stated differently, all calculations presented in this work have been done for the initial system of size $N=2^{17}=$ 131072 and then results have been extrapolated to the limit $N \rightarrow \infty$ using the Shanks method [18].

On the transition line, the normalized cumulant moments $\gamma_{p}^{(z)}$ can be calculated analytically for any $p_{F}$ and $\alpha>-1$. Let us consider first the BS domain. The moments of the multiplicity distribution are $(c f(21)$ and $(22))$ :

$$
<m^{k}>_{N} \sim \frac{1}{p_{F}} \frac{k !\left[a p_{F} \Gamma(\alpha+\tau)\right]^{k}}{\Gamma(k(\tau-1)+\alpha+1)} N^{k(\tau-1)} \psi_{k}
$$


when $N$ is large and $k$ is fixed. Keeping only the leading terms for $N$ large, one gets asymptotically following solutions :

$$
\begin{aligned}
\gamma_{2}^{(z)} & =\frac{1}{p_{F}} \frac{2\left[p_{F} \Gamma(\alpha+\tau)\right]^{2}}{\Gamma(2(\tau-1)+\alpha+1)} \psi_{2}-\psi_{1}^{2} \\
\gamma_{3}^{(z)} & =\frac{1}{p_{F}} \frac{6\left[p_{F} \Gamma(\alpha+\tau)\right]^{3}}{\Gamma(3(\tau-1)+\alpha+1)} \psi_{3}-3 \frac{1}{p_{F}} \frac{2\left[p_{F} \Gamma(\alpha+\tau)\right]^{2}}{\Gamma(2(\tau-1)+\alpha+1)} \psi_{2} \psi_{1}+2 \psi_{1}^{3} \\
\gamma_{4}^{(z)} & =\frac{1}{p_{F}} \frac{24\left[p_{F} \Gamma(\alpha+\tau)\right]^{4}}{\Gamma(4(\tau-1)+\alpha+1)} \psi_{4}-4 \frac{1}{p_{F}} \frac{6\left[p_{F} \Gamma(\alpha+\tau)\right]^{3}}{\Gamma(3(\tau-1)+\alpha+1)} \psi_{3} \psi_{1}+ \\
& +12 \frac{1}{p_{F}} \frac{2\left[p_{F} \Gamma(\alpha+\tau)\right]^{2}}{\Gamma(2(\tau-1)+\alpha+1)} \psi_{2} \psi_{1}^{2}-3\left[\frac{1}{p_{F}} \frac{2\left[p_{F} \Gamma(\alpha+\tau)\right]^{2}}{\Gamma(2(\tau-1)+\alpha+1)} \psi_{2}\right]^{2}-6 \psi_{1}^{4} \\
\ldots & =\ldots
\end{aligned}
$$

, which are valid for $\alpha>-1$ and $p_{F}>1 / 2$. These solutions for $\gamma_{p}^{(z)}$ are independent of the system size $N$ and hence of the average fragment multiplicity $<m>_{N}$ (eq. (19) ), provided this size is large enough. This is a property of distributions obeying the KNO scaling (6) as well as the distributions in the class of Poisson transforms.

One can easily verify that all moments $\gamma_{p}{ }^{(z)}$ are infinite for $p_{F} \rightarrow 1 / 2$, and vanish for $p_{F}=1$. Moreover, $\gamma_{3}{ }^{(z)}$ becomes negative for $p_{F}$ larger than some value which depends on $\alpha$ (provided $\alpha<2$ ). For example, $\gamma_{3}(z)$ for $\alpha=0$ is negative for $p_{F}>0.8856$. The dependence of $\gamma_{3}{ }^{(z)}$ and $\gamma_{4}{ }^{(z)}$ on $p_{F}$ for $\alpha=-1 / 2,0$ and $+1 / 2$ is shown in Figs. 3 and 4 respectively.

B. The marginal case: $p_{F}=1 / 2, \alpha>-1$

The case $p_{F}=1 / 2$ corresponds to the critical point of the FIB branching process. This case has not been solved up to now for general $\alpha$. We present hereafter the exact solution for the remarkable point : $\alpha=0$. To find this solution, one uses the fact that the moments $<m^{k}>_{N}$ depend only on simple combinations of quantities : 


$$
S_{q}(N)=\sum_{j=1}^{N-1} \frac{1}{j^{q}} .
$$

In the expression for the moment $<m^{p}>_{N}$, only the combinations of $S_{q}(N)$ satisfying the relation $q_{1}+q_{2}+\ldots \leq 2 p-1$ are allowed. It is then easy to obtain all solutions for $<m^{k}>_{N}(k=1, \ldots)$ using symbolic operations :

$$
\begin{aligned}
<m>_{N} & =\frac{1}{2} S_{1}(N) \\
<m^{2}>_{N} & =\frac{1}{2} S_{1}(N)-\frac{1}{2} S_{2}(N)+\frac{1}{2} S_{1}^{2}(N)+ \\
& +\frac{1}{6} S_{3}(N)-\frac{1}{4} S_{1}(N) S_{2}(N)+\frac{1}{12} S_{1}^{3}(N) \\
<m^{3}>_{N} & =\frac{1}{2} S_{1}(N)-\frac{3}{2} S_{2}(N)+\frac{3}{2} S_{1}^{2}(N)+2 S_{3}(N)-3 S_{1}(N) S_{2}(N)+ \\
& +S_{1}^{3}(N)-\frac{3}{2} S_{4}(N)+2 S_{1}(N) S_{3}(N)-\frac{3}{2} S_{1}^{2}(N) S_{2}(N)+ \\
& +\frac{3}{4} S_{2}^{2}(N)+\frac{1}{4} S_{1}^{4}(N)+\frac{3}{5} S_{5}(N)-\frac{3}{4} S_{1}(N) S_{4}(N)- \\
& -\frac{1}{2} S_{2}(N) S_{3}(N)+\frac{1}{2} S_{1}^{2}(N) S_{3}(N)-\frac{1}{4} S_{1}^{3}(N) S_{2}(N)+ \\
& +\frac{3}{8} S_{1}(N) S_{2}^{2}(N)+\frac{1}{40} S_{1}^{5}(N) \\
\ldots & =\ldots
\end{aligned}
$$

As in the previously discussed case (sect. 5.A) case, one can compute using these solutions the leading behaviour of the normalized cumulant moments (10):

$$
\gamma_{p} \sim \frac{1}{2 p-1} \ln ^{p-1} N \simeq \frac{2^{p-1}}{2 p-1}\left(<m>_{N}\right)^{p-1}
$$

which diverge when $N \rightarrow \infty$, following the divergence of the average multiplicity (20) when $N \rightarrow \infty$. Hence, the multiplicity distributions in the marginal case $p_{F}=1 / 2$ and $\alpha>-1$ do not belong to the class of the Poisson transforms and do not obey the asymptotic KNO scaling law. 


\section{Cayley fragmentation regime : $p_{F}<1 / 2, \alpha>-1$}

FIB process in this regime is analogous to the invasion percolation on the Cayley tree, because for $p_{F}<1 / 2$ and $\alpha>-1$, the cut-off scale for monomers does not intervene [11] . In the following, we shall call this kind of fragmentation the 'Cayley' or the 'highviscosity' fragmentation. In this regime, the sequential fragmentation process leads naturally to power-law distributions in space and time and is analogous to the SOC phenomenon. In ref. [11], the avalanches were defined in terms of the fluctuating instantaneous dissipation rate :

$$
f(t)=\sum_{k} \chi_{k}(t)
$$

where the summation goes over all clusters in the fragmentation cascade. $f(t)$ is an indicator function of unstable clusters at a time $t \cdot \chi_{k}(t)$ is the characteristic function of the cluster $k$ and equals either 1 , for $t \in\left[t_{k}, t_{k}^{\prime}\right]$ where $t_{k}$ is the time when the cluster $k$ appears and $t_{k}^{\prime}$ is the time when it disappears, or 0 otherwise. The avalanche-size is then defined as the total dissipation :

$$
\hat{s}=\int_{0}^{\infty} f(t) d t=\sum_{k}\left(t^{\prime}{ }_{k}-t_{k}\right) \sim \tilde{m} \tilde{T},
$$

of the fragmentation avalanche. This is just the sum of the lifetimes of all the clusters which have appeared in the sequence of breaks, where $\tilde{m}$ and $\tilde{T}$ are the average multiplicity and the total fragmentation time for a given event respectively. For each value of the fragmentation probability $p_{F}$, FIB process provides a different mean-field realization of a SOC phenomenon with its particular exponents of the power-law spatiotemporal distributions [11]. The relation between the invasion percolation and the SOC phenomenon was noticed independently by Roux and Guyon [20] .

Following (3) and assuming that there exists a limiting stable distribution :

$G_{N}(x) \rightarrow G(x)$ when $N \rightarrow \infty$, one may derive the following equation for the generating function : 


$$
G(x)=\left(1-p_{F}\right)+p_{F} x G^{2}(x) \quad
$$

Solving this equation for $G(x)$, one obtains the multiplicity probability distribution for large enough sizes $N$ :

$$
P_{m}=\frac{(2 m) !}{m !(m+1) !} \frac{a^{m}}{(1+a)^{2 m+1}} \quad(\alpha>-1)
$$

where $a^{-1}=1+\langle m\rangle^{-1}$, as long as :

$$
<m>_{N}=\frac{p_{F}}{1-2 p_{F}}
$$

is positive. Eq. (33) can also be rewritten in the form :

$$
P_{m}=\frac{1}{m+1} \frac{(2 m) !}{m ! m !} p_{F}^{m}\left(1-p_{F}\right)^{m+1} \quad\left(p_{F}<1 / 2, \quad \alpha>-1\right),
$$

which explicitly shows the dependence on the fragmentation probability and hence on $\tau$. This distribution is peaked at $m=0$ and falls down for large multiplicities. As can be seen from (16), the average multiplicity $\left\langle m>_{N}\right.$ for each $p_{F}$ is asymptotically constant and neither depends on the total size $N$ of the fragmenting system, nor on the value of $\alpha$. Obviously, these distributions do not belong to the class of Poisson transform distributions and do not also satisfy the KNO scaling. The independence of functions $\gamma_{p} \equiv f_{p} /\left(f_{1}\right)^{p}$ on the system size is not a sufficient condition for this function to obey the KNO scaling. In any case, the normalized factorial cumulant moments $\gamma_{p} \equiv f_{p} /\left(f_{1}\right)^{p}$ provide valuable informations about the multifragment correlations. We shall return to this problem in chapter 7 when discussing the structure of multifragment correlations. The values of the first few normalized cumulant moments are given by :

$$
\begin{aligned}
\gamma_{2} & =\frac{3-4 p_{F}}{1-2 p_{F}} \\
\gamma_{3} & =6 \frac{\left(1-p_{F}\right)\left(3-4 p_{F}\right)}{\left(1-2 p_{F}\right)^{2}} \\
\gamma_{4} & =6 \frac{35-126 p_{F}+154 p_{F}^{2}-64 p_{F}^{3}}{\left(1-2 p_{F}\right)^{3}} \\
\ldots & =\ldots
\end{aligned}
$$


Similarly as in the BS fragmentation regime, we see once again the divergence of these moments for $p_{F} \rightarrow 1 / 2$. In Fig. 5 we show few examples of the multiplicity distributions for different values of the fragmentation probability : $p_{F}=0.05,0.25,0.45$. If $z \equiv m /<m>$ is large, then these distributions behave like:

$$
P(z)=z^{-3 / 2} \exp (-b z)
$$

where

$$
b=\frac{p_{F}}{1-2 p_{F}} \ln \left[4 p_{F}\left(1-p_{F}\right)\right] \quad,
$$

Note the appearance of a power-law distribution $P(z) \sim z^{-3 / 2}$ when $p_{F} \rightarrow 1 / 2$.

\section{Evaporative fragmentation regime: $p_{F}>0, \alpha<-1$}

The fragmentation kernels in this regime are strongly asymmetric and the preferred splitting at each step in the fragmentation cascade is $:(k) \rightarrow(k-1)+(1)$. This process resembles the evaporation of light fragments (predominantly monomers) from a big cluster and therefore we shall call it the 'evaporative fragmentation regime'. At each step of this process, one branch of the binary fragmentation dies out almost surely. The dominance of only one fragmentation branch associated with a big cluster, leads to an approximate $N$ - independence of the average multiplicity (see Fig. 6). Hence, also in this domain the multiplicity probability distributions are neither Poisson transforms, nor they obey the KNO scaling.

The limiting case $\alpha=-\infty$ is particularly interesting as in this case at each step of the fragmentation one monomer is separated from the large cluster. The generating function of the probability distribution for $\alpha=-\infty$, which can be derived from eq. (3), satisfies the recurrence relation :

$$
G_{N}(x) \sim 1-p_{F}+p_{F} x G_{N-1}(x)
$$

which yields : 


$$
G_{N}(x) \sim \frac{1-p_{F}}{1-p_{F} x}
$$

The probability distribution in this case :

$$
P_{N}[m] \sim\left(1-p_{F}\right) p_{F}^{m}
$$

is a special case of a gamma distribution :

$$
P_{k}(z)=\frac{\gamma^{k}}{\Gamma(k)} \exp (-\gamma z) z^{k-1}
$$

in the variable $z=m /\langle m\rangle$, with :

$$
\gamma=\frac{p_{F}}{1-p_{F}} \ln \left(\frac{1}{p_{F}}\right)
$$

and :

$$
<z>=\frac{p_{F}}{\gamma^{2}}=\frac{k}{\gamma}
$$

$\gamma$ equals 1 in the limit $p_{F} \rightarrow 1$, and one recovers from (38) the negative binomial distribution :

$$
P_{k}(z)=\frac{k^{k}}{\Gamma(k)} \exp (-k z) z^{k-1}
$$

The Bose-Einstein distribution corresponds to $k=1$.

The multiplicity distributions in the evaporative phase for several values of the parameter $p_{F}$ are shown in Fig. 7 for $\alpha=-5 / 2$ and in Fig. 8 for $\alpha=-\infty$. The multiplicity distributions exhibit strong dependence on $\alpha$. Close to the border line between BS and evaporative fragmentation regimes (see Fig. 1), the multiplicity distributions in the evaporative phase exhibit certain similarities with those in the BS phase (see Fig. 2) and are different from the distributions in the limit $\alpha \rightarrow-\infty$.

In Figs. 9 and 10 we show the dependence of the normalized cumulant moments of order $p=3$ and 4 on the fragmentation probability $p_{F}$ for three different values of $\alpha:-5 / 2,-7 / 2$ (the solid lines) and $-\infty$ (the dashed line). In the latter case, $\gamma_{p}$ is 
independent of $p_{F}$ and equals $(p-1)$ !, as for the Bose-Einstein distribution. One should stress that $\gamma_{p}$ cannot be calculated using the integral form of the relation (10) with the limit (38) for $f(z)$, because $<m>_{N}$ is independent of $N$ and hence the multiplicity distributions in the evaporative phase do not belong to the class of Poisson transforms. Consequently, $\gamma_{p}$ in Figs. 9 and 10 are calculated directly using $f_{p}$ 's (11). The value of $\gamma_{p}$ for $\alpha=-\infty$ suggest that we are dealing with the Bose-Einstein distribution whereas, in fact, it is the gamma distribution which approaches the Bose-Einstein distribution asymptotically when $p_{F} \rightarrow 1$. Nevertheless, as can be seen in Fig. 11 where $\gamma_{3} /\left(2 \gamma_{2}{ }^{2}\right)$ is plotted versus $p_{F}$ for different $\alpha$, the relations between moments $\gamma_{p}$ of different rank for those different distributions in the evaporative phase are 'similar' to the corresponding relations for the negative binomial distribution. For $\alpha=-\infty$ (the dashed line), this quantity equals 1 , exactly as for the negative binomial distribution. The curves $\gamma_{3} /\left(2 \gamma_{2}{ }^{2}\right)$ vs. $p_{F}$ calculated for different $\alpha$ 's $(\alpha \neq-\infty)$, all converge to 1 in the limit $p_{F} \rightarrow 1$.

\section{BRAND - SCHENZLE FRAGMENTATION REGIME AS A MULTIPLICATIVE STOCHASTIC PROCESS}

The generic statistical framework for the appearance of the KNO scaling in the binary fragmentation process, is provided by the cascade equations (1). They can be written also in the form of the rate equations:

$$
\begin{aligned}
\frac{\partial P_{N}[m ; t]}{\partial t} & +I_{N} P_{N}[m ; t]= \\
& =\sum_{j=1}^{N-1} F_{j, N-j}\left(\sum_{m^{\prime}=0}^{m-1} P_{j}\left[m^{\prime} ; t-t^{\prime}\right] P_{N-j}\left[m-m^{\prime}-1 ; t-t^{\prime}\right]-P_{N}[m ; t]\right)+ \\
& +I_{N} \delta(m),
\end{aligned}
$$

which are basically non-linear. Rate equations provide a traditional context for number evolutions [21]. The effect of mode-mode coupling can be often represented as a noise or 
fluctuating force, acting on the chosen mode. These fluctuations arise from the elimination of the irrelevant microscopic degrees of freedom in favour of a small number of macroscopic variables. In this way, one arises at the Langevin formulation of the initial multidimensional problem in which macroscopic variables $\{\vec{z}\}$ are driven by the fluctuating force $F(t)$ :

$$
\frac{d}{d t} z_{i}=\Gamma_{i}(\{\vec{z}\})+G(\{\vec{z}\}) F(t)
$$

The stochastic process (41) is called additive if $G$ is independent of $\{\vec{z}\}$, otherwise it is called the multiplicative stochastic process.

The multiplicity distributions generated by the rate equations (40), when expressed in the KNO variable, are identical in the whole BS domain to the special solution of the onedimensional, nonlinear stochastic processes with multiplicative fluctuations :

$$
\frac{d}{d t} z=D z-B z^{1+\gamma}+z F
$$

which was studied by Brand and Schenzle [19] in connection with certain stochastic processes in non-linear optics and chemical reactions. Solution of this equation include also many analytic functions proposed to describe multiplicity distributions in $p p$ and $e^{+} e^{-}$collisions $[23]$. In the above equation, $F$ represents a Gaussian white noise :

$$
<F(t) F\left(t^{\prime}\right)>=Q \delta\left(t-t^{\prime}\right)
$$

and the variable $z$ should be identified with the KNO scaled variable $m /<m>$. Associated Focker - Planck equation :

$$
\frac{\partial}{\partial t} f(z, t)=-\frac{\partial}{\partial z}\left[\left(D z-B z^{1+\gamma}+\frac{1}{2} Q z\right) f(z, t)\right]+\frac{Q}{2} \frac{\partial^{2}}{\partial z^{2}}\left(z^{2} f(z, t)\right)
$$

permits an interpretation of the FIB process in the BS fragmentation regime in terms of the generalized diffusion process. The parameters $(B, D, \gamma)$ of the BS equation $(42),(44)$ can be expressed by a unique parameter $\tau$ of the power-law fragment-size distribution : 


$$
\begin{aligned}
\frac{2 B}{Q} & =(\tau-1)^{\frac{\tau-1}{2-\tau}} \\
\frac{2 D}{Q} & =\frac{1}{2(2-\tau)} \\
\gamma & =\frac{1}{2-\tau} .
\end{aligned}
$$

Thus, the multiplicity evolution in the BS fragmentation domain is a special case of the BS stochastic multiplicative equation with the parameters $B(\tau), D(\tau)$ and $\gamma(\tau)(1 \leq \tau \leq 2)$, defining a line in the manifold $\{B, D, \gamma\}$. The KNO scaling function (26) depends only on $\tau(1<\tau<2$ following eq. (14) ) and, hence, the kinetic aspects of the FIB process which are contained in the fragmentation and inactivation functions cannot be studied unambiguously. Formally, parameters $B, D, \gamma$ can be identified with parameters of the fundamental Hamiltonian of the system as it was demonstrated for example in the laser model [24]. The FIB model with $\tau=3 / 2$, corresponding to a special case of the BS equations with $\gamma=2$, leads indeed to the laser model [24]. For $\gamma=1, B=D$ and $2 D / Q=k$, the BS equations are equivalent to the linear rate equations for the multiplicity evolution [17] :

$$
\frac{d}{d t} P_{m}=(m-1) \lambda P_{m-1}-m \lambda P_{m} \quad\left(P_{0}=0\right)
$$

for which $<m>(t)=k e^{\lambda t}$. The solution of this equation has the same scaling limit (39) as the negative binomial distribution with :

$$
k=<m>(t) e^{-\lambda t}=\frac{2 D}{Q}
$$

The FIB process (40) is compatible with the linear rate equations only in the limit when $p_{F} \rightarrow 1 / 2$, though the particular solution for $\gamma=1$ (eq. (46)) is not satisfied by the FIB model in the BS regime for which $B \neq D$. 


\section{THE STRUCTURE OF THE HIGHER ORDER CUMULANT CORRELATIONS}

The $n$-fragment correlation function consists mainly of statistical combination of lower

order correlations. In order to study genuine $n$-fragment correlations, one has to define the $n$-fragment cumulant $K_{n}\left(y_{1}, \ldots, y_{n}\right)$, which enters into the expression for $\rho_{n}$ together with cumulants of the order lower than $n$. The first few densities are :

$$
\begin{aligned}
\rho_{2}(1,2)= & \rho_{1}(1) \rho_{1}(2)+K_{2}(1,2) \\
\rho_{3}(1,2,3)= & \rho_{1}(1) \rho_{1}(2) \rho_{1}(3)+\sum \rho_{1}(i) K_{2}(j, k)+K_{3}(1,2,3) \\
\rho_{4}(1,2,3,4)= & \rho_{1}(1) \rho_{1}(2) \rho_{1}(3) \rho_{1}(4)+\sum \rho_{1}(i) \rho_{1}(j) K_{2}(k, l) \\
& +\sum \rho_{1}(i) K_{3}(j, k, l)+\sum K_{2}(i, j) K_{2}(k, l) \\
& +K_{4}(1,2,3,4)
\end{aligned}
$$

and so on, where the sums are taken over all permutations of $\{1, \ldots, n\}$ without the transposition inside the factors of the sums. The $n$-fragment distribution densities in (47) are related to the $n$-fragment inclusive cross sections :

$$
\rho_{n}\left(y_{1}, \ldots, y_{n}\right)=\frac{1}{\sigma_{I}} \frac{d^{n} \sigma}{d y_{1} \ldots d y_{n}} \quad n=1,2 \ldots
$$

where $\sigma_{I}$ is the total inelastic cross section. The $n$-fragment cumulant measures the statistical dependence of the whole $n$-fragment set. The $n$-fragment cumulant is zero, if anyone of the $n$ fragments is independent of the others. The second order cumulant is equivalent to the two-fragment correlations, but already at the three-fragment level the study of the cumulant distribution requires the subtraction of the combinatorial part from the lower order correlations from the genuine three-fragment correlation function.

The factorial moments arise from the integration of the corresponding particle distribution densities over a domain $\Omega$ : 


$$
\begin{aligned}
& \tilde{F}_{1}=<m>_{\Omega}=\int_{\Omega} d y \rho_{1}(y) \\
& \ldots=\ldots \\
& \tilde{F}_{k}=<m(m-1) \ldots(m-k+1)>_{\Omega}=\int_{\Omega} d y_{1} \ldots \int_{\Omega} d y_{k} \rho_{k}\left(y_{1}, \ldots, y_{k}\right) \\
& \ldots=\ldots
\end{aligned}
$$

The choice of $\Omega$ depends on the physical problem considered. For example, in relativistic heavy-ion collisions or high energy hadronic collisions, domain $\Omega$ refers usually to the kinematic variables such as the longitudinal (rapidity) or transverse momenta [25]. At lower energies, in the total charge fragmentation regime $(E / A \simeq 5-7 \mathrm{MeV})$, this domain was also identified with the range of masses (charges) of the fragments [26,27]. In photoelectron count experiments, $\Omega$ refers to the arrival time-interval of photons exciting electrons in the detector.

Similarly as for the factorial moments (49), the factorial cumulants (11) arise in the integration of the multifragment (multiparticle) correlation function $K_{n}\left(y_{1}, \ldots, y_{n}\right)$ over a domain $\Omega$ of the studied distribution. The equations (11) relating factorial moments with factorial cumulant moments are exact independently of the functional form of the underlying correlation function. Given $\tilde{F}_{2}$, we may compute $f_{2}$ and insert this into the $\tilde{F}_{3}$ identity in (11). Measuring $\tilde{F}_{3}$ in a domain $\Omega$ gives then $f_{3}$, and so forth. Hence, if the experimental statistical accuracy allows, one has access to the sequence of cumulant moments and hence to the genuine, higher order fragment correlations. If all cumulant moments of order three and higher are zero then only two-fragment correlations are present in the system. Similarly, if only two- and three-particle correlations are present, the corresponding $f_{3}$ term would be present but all cumulant factorial moments of order higher than three would be identically zero. The structure of higher order cumulants was addressed in the context of high energy nuclear collisions where for certain reactions the cumulants of order 3 and higher were found to be zero [28]. This yields a particularly simple correlation scheme for those multihadron production processes. In the FIB model, this correlation scheme is nowhere exactly realized as show the analytical results in eqs. (28), (30) and (36) for the BS domain, the marginal 
case $\left(p_{F}=1 / 2, \alpha>-1\right)$ and the Cayley domain respectively. The numerical results for the evaporative fragmentation regime, shown in Figs. $8-10$, confirm this finding as well. However, when $p_{F}$ is close to 1 in the BS fragmentation domain, the higher order cumulant moments approach zero much faster than does the second order cumulant moment so the leading behaviour of the binary fragmentation process can be closely approximated neglecting fragment-fragment correlations of order higher than two.

For other types of high energy reactions, the linked-pair approximation (LPA) of the higher order cumulants, inspired by the structure of the galaxy distribution in the universe [29], was applied to fit the data [28,30]. In the framework of the LPA, the two-fragment cumulant correlations provide the building blocks of the higher order cumulants [28,31] , which are built up as sums of products of linked two-particle cumulants :

$$
K_{p}(1,2 \ldots p)=A_{p} \sum_{p e r m} \prod^{p-1} K_{2}(1,2)
$$

Assuming the translational invariance and evaluating cumulant moments in the strip approximation [28], produces normalized factorial cumulant moments obeying :

$$
\frac{f_{p}}{\left(f_{1}^{p}\right)} \equiv \gamma_{p}=A_{p}\left(\gamma_{2}\right)^{p-1}=A_{p}\left(\frac{f_{2}}{\left(f_{1}\right)^{2}}\right)^{p-1}
$$

Consequently :

$$
\frac{A_{p}}{A_{p-1}}=\frac{\gamma_{p}}{\gamma_{p-1} \gamma_{2}}=\left(\frac{f_{p}}{f_{p-1}}\right)\left(\frac{f_{1}}{f_{2}}\right)
$$

and :

$$
A_{p}=\gamma_{p}\left(\gamma_{2}^{1-p}\right)
$$

The validity of LPA can also be tested through various scaling laws such as [32] :

$$
\frac{f_{p}}{f_{q}}=\frac{A_{q}}{A_{p}}\left(\frac{f_{2}}{f_{1}}\right)^{q-p} .
$$

The hierarchical amplitudes $A_{p}$ in the above formulas are free parameters to be determined from the fit to the data. For the LPA approximation to be valid, the coefficients $A_{p}$ or 
equivalently $\gamma_{p}$ should be independent of the fragment multiplicity $<m>_{N}$ and hence of the size $N$ of the fragmenting object. This is the case in all domains of the multiplicity distributions for the FIB process at the transition line with a notable exception for the marginal case $p_{F}=1 / 2$.

The hierarchical structures for higher order cumulant correlations $(50)$ have been used to fit the correlation of galaxies and higher-order correlations in hadronic multiparticle data. Numerous phenomenological hierarchical models which posses this linking scheme for correlation functions have been constructed for that purpose [33]. The difference between them lies in the pattern of the $A_{p}$ coefficients which measure the amplification of higher order correlations.

In the Cayley domain of the FIB process, $A_{3}$ starts from 2 for $p_{F}=0$ and approaches 3 when $p_{F} \rightarrow 1 / 2$. In the limit $p_{F} \rightarrow 1 / 2$ of the BS fragmentation regime, the coefficient $A_{3}$ approaches $3 / 2$ and decreases to $-\infty$ when $p_{F} \rightarrow 1$. Thus, we have a discontinuous change of the high order correlations when passing from Cayley domain to BS domain through the critical point $p_{F}=1 / 2$ of the branching process. The marginal case ( $p_{F}=$ $1 / 2, \alpha>-1)$ is particularly interesting because the coefficients $A_{p}$ are independent of $N$ eventhough all $\gamma_{p}$ depend on $N$ explicitly. One should remind that the multiplicity distributions in this case do not belong to the class of Poisson transforms and do not satisfy the KNO scaling. In this special case, $A_{3}$ equals $9 / 5$ for $\alpha=0$.

In the BS domain of the FIB fragmentation process, we have succeeded to calculate the dependence of $A_{3}$ on $p_{F}$ analytically. At $p_{F} \rightarrow 1 / 2$ the coefficient $A_{3}$ equals $3 / 2$, independently of $\alpha$, and decreases monotonously with increasing $p_{F}$. For $\alpha=0$ it becomes negative for $p_{F}>0.8856$ and diverges to $-\infty$ when $p_{F} \rightarrow 1$. The precise value of $A_{3}$ in the BS regime depends strongly on both $\alpha$ and $p_{F}$ and one may hope to learn about the details of the rate functions from the detailed knowledge of $A_{p}$ 's. On the contrary, the hierarchical amplitudes are strictly independent of $\alpha$ in the Cayley regime. In the evaporative phase, amplitudes $A_{3}$ are only weakly dependent on both $\alpha$ and $p_{F}$. In the limiting case $\alpha=-\infty$ (the dashed line), $A_{3}$ in the evaporative phase equals 
2 independently of $p_{F}$. Qualitatively similar behaviour is seen also for $A_{\mathbf{4}}$. Figs. 12 and 13 show the dependence of the hierarchical amplitudes $A_{3}$ and $A_{4} / A_{3}$ on the fragmentation probability $p_{F}$ in different fragmentation regimes on the transition line.

In conclusion, the FIB process on the transition line has the hierarchical structure of higher order cumulants and obey the LPA everywhere. In the Cayley and evaporative fragmentation domains, this structure is somewhat trivial as the mean multiplicity of fragments $<m>_{N}$ is approximately independent of the system size and changes with the parameters $\left\{p_{F}, \alpha\right\}$ only. In these two domains, $A_{p}$ are either strictly independent of $\alpha$ (the Cayley domain) or the dependence on $\alpha$ is very weak (the evaporative domain). The hierarchical amplitudes $A_{p}$ are discontinuous while passing from the Cayley fragmentation regime to the BS regime through the critical point of the FIB branching process. Thus, exact empirical knowledge of those amplitudes as a function of $p_{F}$ permits, in principle, to detect the change in the structure of higher order multifragment correlations associated with passing through the critical point of the associated branching process.

\section{CONCLUSIONS}

In this work we have analyzed in details the properties of the multiplicity probability distributions as obtained in the non-equilibrium sequential binary fragmentation model with the inhibition. We have restricted our analysis to the transition line between the $\infty$-cluster and shattering phases of the binary fragmentation. In this region, the FIB process is selfsimilar at all scales until the low-mass cutoff for monomers, i.e. the fragmentation probability does not depend on the size of the fragmenting cluster for all fragments with masses $s>1$. In our earlier works, we have studied the asymptotic cluster-size distribution which at the transition line is given by the power-law $n_{s} \sim s^{-\tau}$ with $\tau \leq 2$. In this region, FIB model describes well the fragment-size distribution and all charge-fragment correlations in the heavy-ion collisions at intermediate energies [34]. Other statistical approaches have been also tried successfully [35] so the supplementary information contained in the multiplicity 
distributions and cumulant correlations is strongly needed.

In this work we have emphasized the intrinsic properties of classes of probability distributions of the critical FIB process in order to see clearly those features not tied to a specific conditions of the kinetic evolution. We believe that this will help in correlating theoretical fragmentation scenarios with experimental results. One should however remember that multiplicity distributions in different domains at the transition line depend principally on the exponent $\tau$ and do not allow for an unambiguous determination of the fragmentation and inactivation kernels. These ambiguities should be strongly underlined in order to discourage premature conclusions.

Eventhough the KNO scaling appears dethroned as a fundamental symmetry of the $S$ matrix at ultrarelativistic energies, nevertheless it should be remembered that much of the energy redundancy is removed by plotting the data in the KNO way. It is then interesting to notice that the KNO scaling appears from our studies as a fundamental property of the critical binary fragmentation process, whenever the average fragment multiplicity $\langle m\rangle_{N}$ depends on the initial system size $N$, i.e. in its 'low-viscosity'- $\left(p_{F}>1 / 2, \alpha>-1\right)$, or BSphase and is absent everywhere outside of the transition line [4]. The appearance of the KNO scaling is hence related to the second-order phase transitions associated with breaking the initial system, characterized by a 'large' scalar quantity (e.g. energy, mass, charge, etc.) and called its 'mass', into a dust fragments each one having only an infinitesimal portion of the initial 'mass'. In this sense the KNO scaling is not only a property of certain relativistic field theories but more generally it appears as a property of the critical fragmentation which can be realized both in quantum systems as well as in the macroscopic classical objects. This new general foundation of the KNO scaling opens a possibility for its finding in many fragmentation processes in nature.

In order to analyze the higher order correlations in the multifragment (multiparticle) distributions one has to recognize that the density correlations contain usually lower order background correlations. These can be conveniently removed using the cumulant correlation functions (47). The statistical independence of any $y_{i}$ in $K_{p}\left(y_{1}, \ldots, y_{p}\right)$ results in factor- 
ization of the $\rho_{p}$ densities and vanishing cumulant. Hence, the cumulants $K_{p}$ are key quantities to be produced by theoretical models of the fragmentation. Following the linked pair ansatz (50), the high order cumulants can be expressed in terms of the cumulants of order two. We have found that the critical FIB process which obeys the KNO scaling of the multiplicity probability distributions, is characterized also by the appearance of the hierarchical structure of the higher order correlations. This particular structure for higher order correlations is absent in both $\infty$-cluster and shattering phases [4]. Up to now, no convincing explanation for the hierarchical structures of the multifragment (multiparticle) correlation functions have been put forward, although Peebles [36] has shown that a random fractal cascade process could have this property. In view of the above results one is tempted to look for the justification of its ubiquity in nature in the ubiquity of the shattering transition. Curiously, the same correlation structures describe galaxy correlations and phase-space correlations in the multiparticle distributions in ultrarelativistic collisions. 


\section{REFERENCES}

[1] R. Botet and M. Ploszajczak, Phys. Rev. Lett. 69, 3696 (1992); Phys. Lett. B312, 30 (1993).

[2] R. Botet and M. Ploszajczak, J. of Mod. Phys. E3, 1033 (1994).

[3] This assumption can be removed if necessary and one can easily consider ternary or even higher order fragmentations as well as for example the statistical distribution of fragment multiplicity at each fragmentation.

[4] R. Botet and M. Ploszajczak, to be published.

[5] This rule does not apply only to the monomers which are not allowed to break up.

[6] In general, one branching process may trigger another one. To take these correlations into account would in principle require the multidimensional generalization of the FIB model. Assuming the direction of this triggering to be random, the critical dimension is $D=4$ [7-9], above which the scaling exponents in the generalized FIB model can be derived from a single FIB branching process as discussed in this work.

[7] N. Jain and S. Orey, Isr. J. Math 6, 379 (1968).

[8] S.P. Obukhov, in Random Fluctuations and Pattern Growth: Experiments and Models, edited by H.E. Stanley and N. Ostrowsky, NATO Advanced Study Institutes, Ser. C, Vol. 157 (Kluwer, Dordrecht, 1988).

[9] P. Alstrom, Phys. Rev. A38, 4905 (1988).

[10] P. Bak, C. Tang and K. Wiesenfeld, Phys. Rev. Lett. 59, 381 (1987); Phys. Rev. A38, 364 (1988).

[11] R. Botet and M. Ploszajczak, Physica A223, 7 (1996).

[12] This result is not general. In particular, the scaling form of the mass distribution results from the homogeneity of kernels. For another homogeneous choice : $F_{i, j}=\frac{1}{2}\left(i^{2 \alpha}+\right.$ 
$\left.j^{2 \alpha}\right), I_{k}=I_{1} k^{\beta}$, which could be relevant for the binary approximation to the spinodal instabilities of the Fermi liquid [13], results are very similar. In particular, $\tau$ is always larger than 2 in the shattered phase.

[13] R. Botet and M. Ploszajczak, Soft Physics and Fluctuations, Proc. of the Cracow Workshop on Multiparticle Production, Cracow, Poland 4 - 7 May 1993, edited by A. Bialas, K. Fijalkowski, K. Zalewski and R.C. Hwa (World Scientific Publ. Co., 1994).

[14] Z. Koba, H.B. Nielsen and P. Olesen, Nucl. Phys. B40, 317 (1972).

[15] N.G. Antoniou, A.I. Karanikas and S.D.P. Vlassopoulos, Phys. Rev. D29, 1470 (1984); ibid. Phys. Rev. D14, 3578 (1976).

[16] For the extensive discussion of the Poisson transforms and their application in different domains of physics, reader is referred to the review by Carruthers and Shih [17] .

[17] P. Carruthers and C.C. Shih, Int. J. Mod. Phys. A2, 1447 (1987).

[18] C.M. Bender and S.A. Orszag, Advanced Mathematical Methods for Scientists and Engineers, McGraw \& Hill, 1978.

[19] A. Schenzle and H. Brand, Phys. Rev. A20, 1628 (1979).

[20] S. Roux and E. Guyon, J. Phys. A22, 3693 (1989).

[21] In the strong interaction physics, they have been discussed extensively as approximation to the QCD branching process [22] . See also ref. [17] for the list of recent applications.

[22] A. Giovannini, G.C. Montovani and S.P. Ratti, Riv. del Nuovo Cimento 2, 1 (1979); C.C. Shih, Phys. Rev. D33, 3391 (1986).

[23] S. Barshay, Phys. Lett. 116B, 193 (1982);

Chou Kuang-chao, Liu Lian-sou and Meng Ta-chung, Phys. Rev. D28, 1080 (1983).

[24] V. DeGiorgio and M.O. Scully, Phys. Rev. A2, 1170 (1970). 
[25] P. Bożek, M. Ploszajczak and R. Botet, Phys. Rep. 252, 103 (1995).

[26] M. Ploszajczak and A. Tucholski, Phys. Rev. Lett. 65, 1539 (1990);

P. Bożek, M. Ploszajczak and A. Tucholski, Nucl. Phys. A539, 693 (1992).

[27] R. Botet and M. Ploszajczak, in Topics in Nuclear and Astro-Nuclear Physics, edited by Z. Sujkowski and J. Skalski, Proc. of the XXIII Mazurian Lakes Summer School Piaski, Poland, August 18-28, 1993, Acta Phys. Polonica B25, 353 (1994).

[28] P. Carruthers and I. Sarcevic, Phys. Rev. Lett. 63, 1562 (1989).

[29] E.J. Groth and P.J.E. Peebles, Ap. J. 217, 285 (1977).

[30] E.A. de Wolf, Acta Phys. Polonica, B21, 611 (1990).

[31] P. Carruthers, H.C. Eggers, Q. Gao and I. Sarcevic, Int. J. Mod. Phys. A6, 3031 (1991).

[32] S. Hegyi, Phys. Lett. B327, 171 (1994).

[33] J.N. Fry, Ap. J. (Letters) 277, L5 (1984); Ap. J. 279, 499 (1984);

W.C. Saslaw and A.J.S. Hamilton, Ap. J. 276, 13 (1984);

R. Schaeffer, Astr. Ap. 144, L1 (1985);

P. Carruthers and C.C. Shih, Phys. Lett. 127B, 242 (1983).

[34] R. Botet and M. Ploszajczak, Phys. Lett. B312, 30 (1993).

[35] A.S. Botvina and I.N. Mishustin, Phys. Lett. B294, 23 (1992);

P. Kreutz et al. (ALADIN Coll.), Nucl. Phys. A556, 672 (1993).

[36] P.J.E. Peebles, The Large Scale Structure of the Universe, Princeton U.P., Princeton, NJ (1980). 


\section{Figure captions}

\section{Fig. 1}

The diagram $p_{F}-\alpha$ showing different multiplicity domains at the transition line of the FIB process. The dashed line represents the line $\tau=3 / 2$ separating two phases : $\hat{z}_{0}=0$ and $\hat{z}_{0}>0$, in the Brand-Schenzle fragmentation regime.

\section{Fig. 2}

Typical multiplicity distributions in the Brand-Schenzle fragmentation regime are plotted in the $\mathrm{KNO}$ variables for various values of $\tau$ in the two phases: $\hat{z}_{0}=0 \quad(\tau \leq 3 / 2)$ and $\hat{z}_{0}>0 \quad(\tau>3 / 2)$.

\section{Fig. 3}

The normalized cumulant moment $\gamma_{3}{ }^{(z)}$ as a function of $p_{F}$ in the Brand-Schenzle fragmentation regime for $\alpha=-1 / 2,0$ and $+1 / 2$.

\section{Fig. 4}

The same as in Fig. 3 but for $\gamma_{4}^{(z)}$.

\section{Fig. 5}

Typical multiplicity distributions in the Cayley fragmentation regime (eq. (35)) for various values of the fragmentation probability : $p_{F}=0.05$ (the long-dashed line), 0.25 (the solid line) and 0.45 (the short-dashed line), are plotted in the KNO variables.

\section{Fig. 6}

Dependence of the average fragment multiplicity $\langle m\rangle_{N}$ on the size of the fragmenting system $N$ in the evaporative fragmentation regime is plotted for $p_{F}=0.25,0.5,0.75$ and for $\alpha=-3 / 2$ (the solid line) and $\alpha=-7 / 2$ (the dashed line). 


\section{Fig. 7}

Typical multiplicity distributions in the evaporative fragmentation regime are plotted in the KNO variables for various values of the fragmentation probability : $p_{F}=0.5,0.75,0.9$ and for $\alpha=-5 / 2$. The simulations correspond to $10^{6}$ fragmentations of the system with the initial size $N=1024$.

Fig. 8

Typical multiplicity distributions in the evaporative fragmentation regime are plotted in the KNO variables for various values of the fragmentation probability : $p_{F}=$ $0.25,0.5,0.75,0.9$ and for $\alpha=-\infty$ (eq. (38)).

\section{Fig. 9}

The normalized cumulant moments $\gamma_{3}$ as a function of $p_{F}$ in the evaporative fragmentation regime for $\alpha=-5 / 2,-7 / 2$ (the solid lines) and $-\infty$ (the dashed line).

Fig. 10

The same as in Fig. 9 but for $\gamma_{4}$.

Fig. 11

The ratio of the normalized cumulant moments $\gamma_{3} /\left(2 \gamma_{2}{ }^{2}\right)$ in the evaporative fragmentation regime is plotted versus $p_{F}$ for different values of $\alpha$. The dashed line corresponds to $\alpha=-\infty$.

Fig. 12

The dependence of the hierarchical amplitude $A_{3}$ on the fragmentation probability $p_{F}$ in different domains at the transition line of the FIB model. 
Fig. 13

The same as in Fig. 12 but for $A_{4} / A_{3}$. 


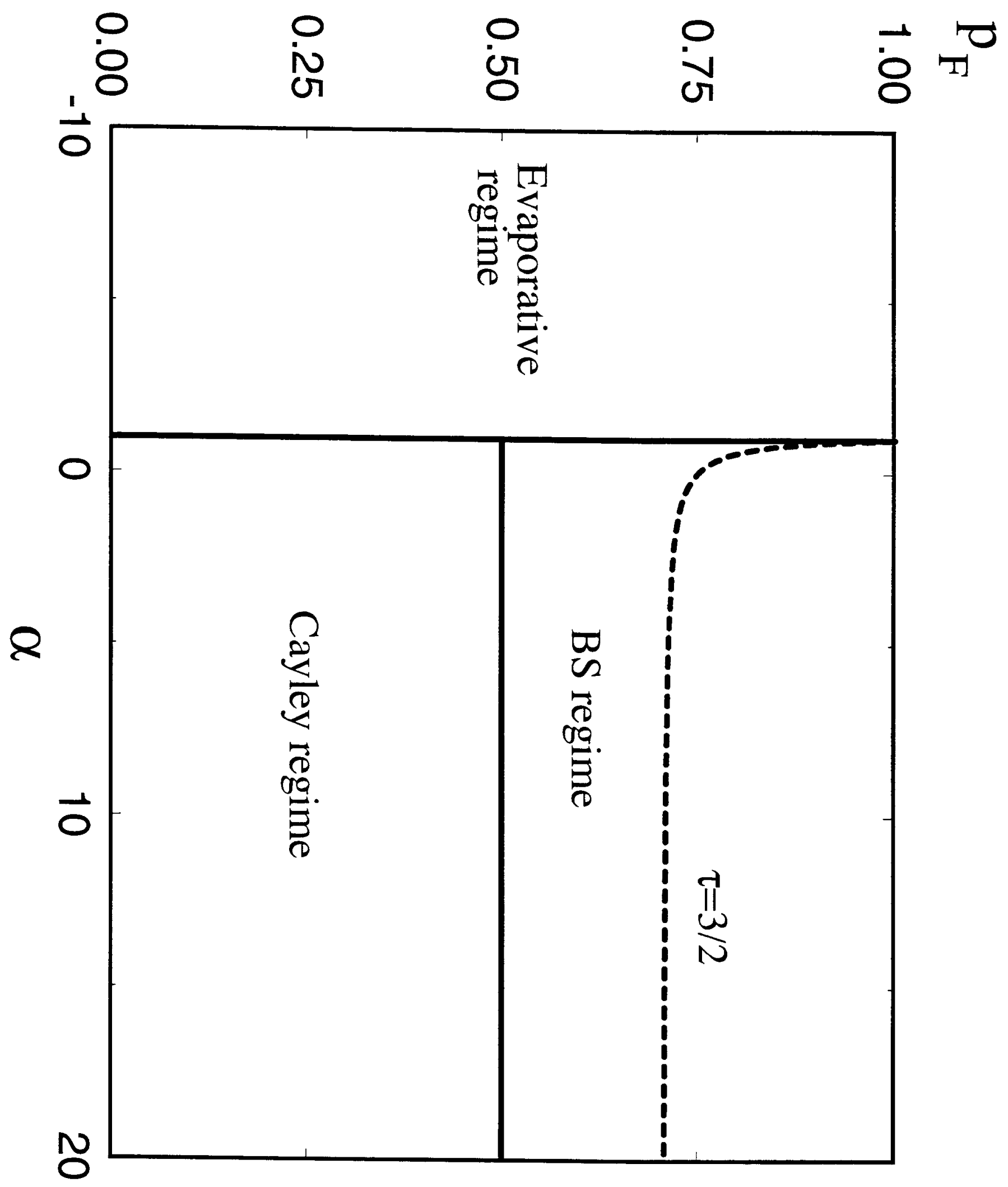

Fig. 1 




Fig. 2 


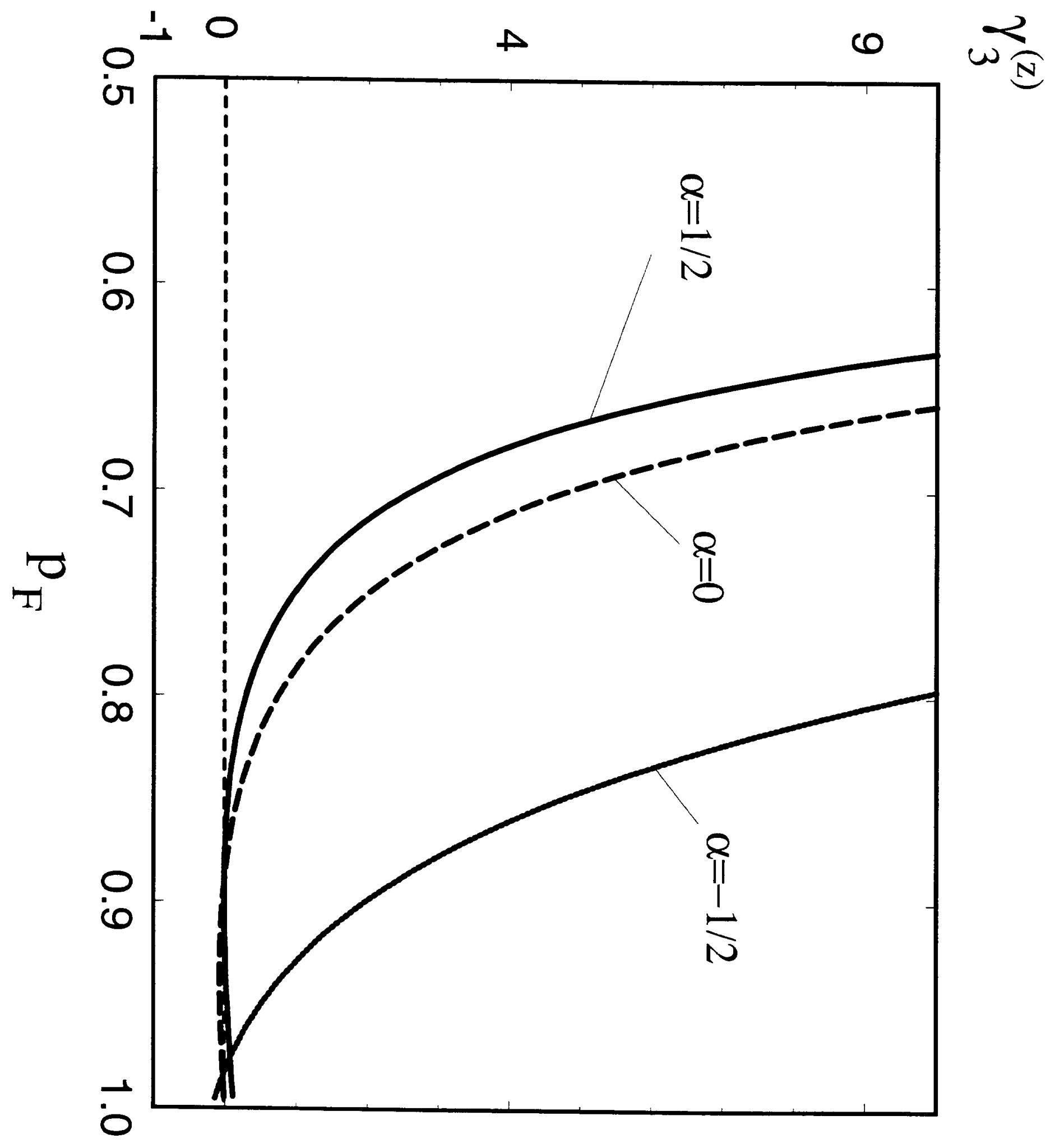

Fig. 3 


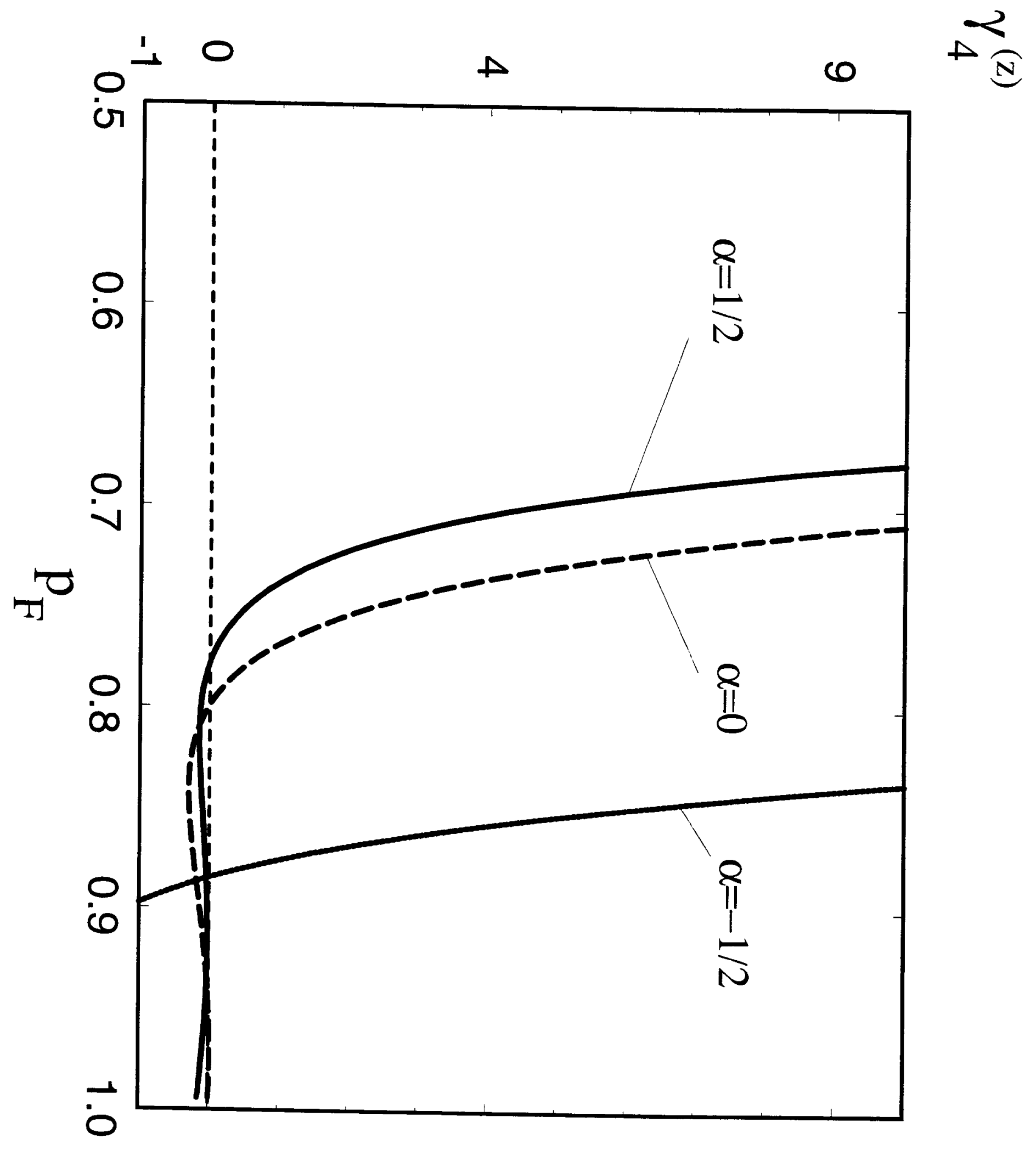

Fig. 4 


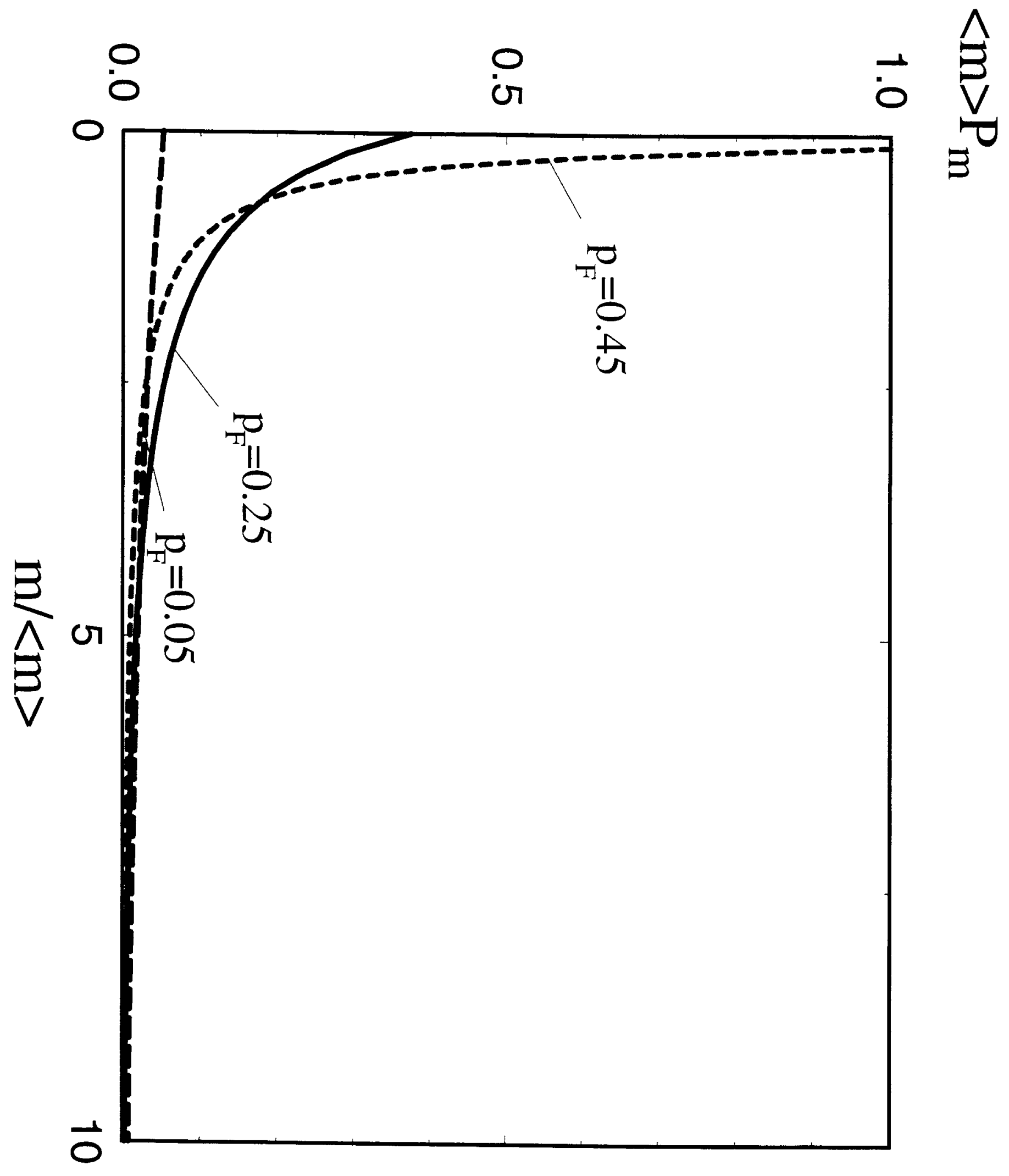

Fig. 5 


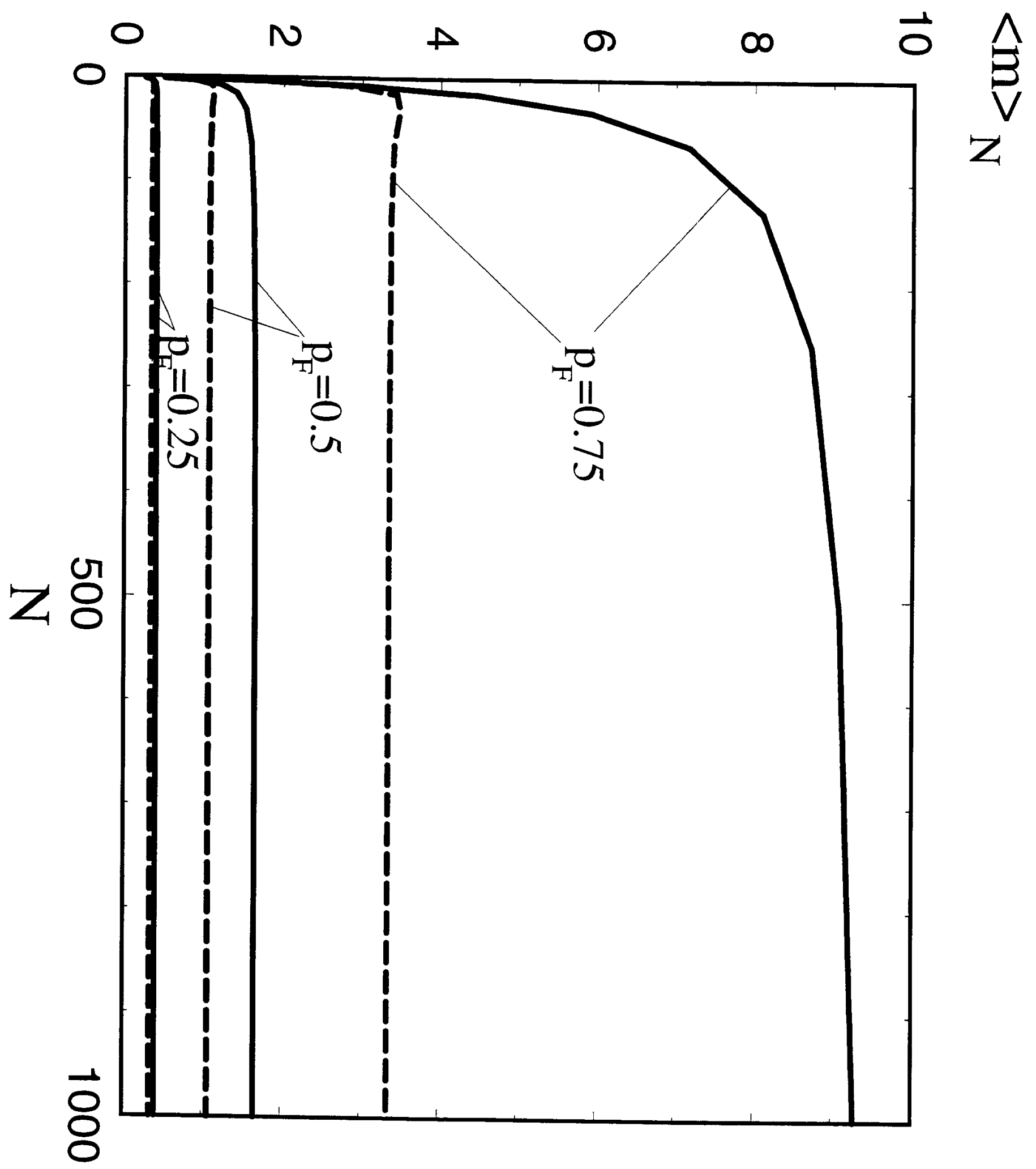

Fig. 6 


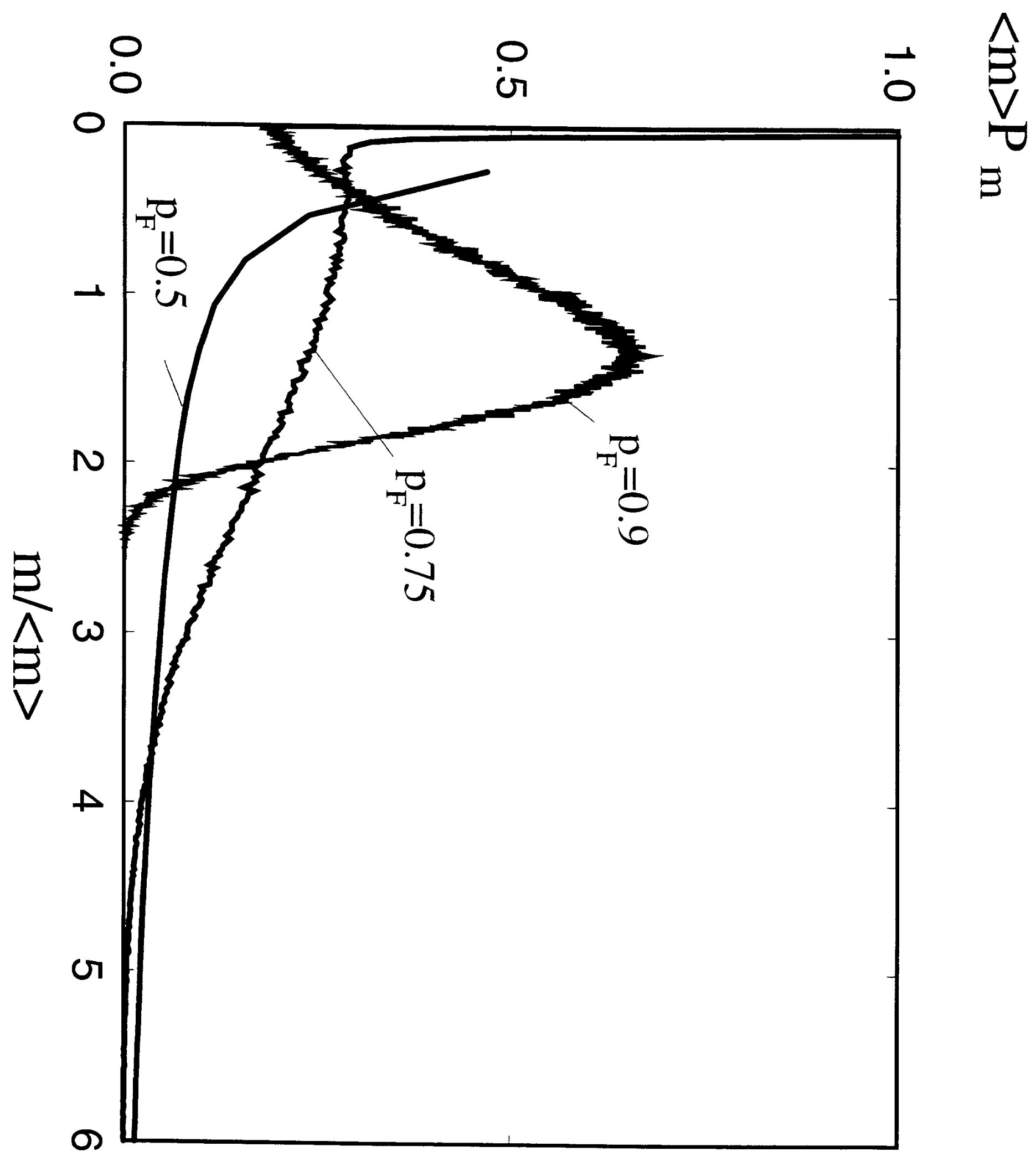

Fig.7 




Fig. 8 


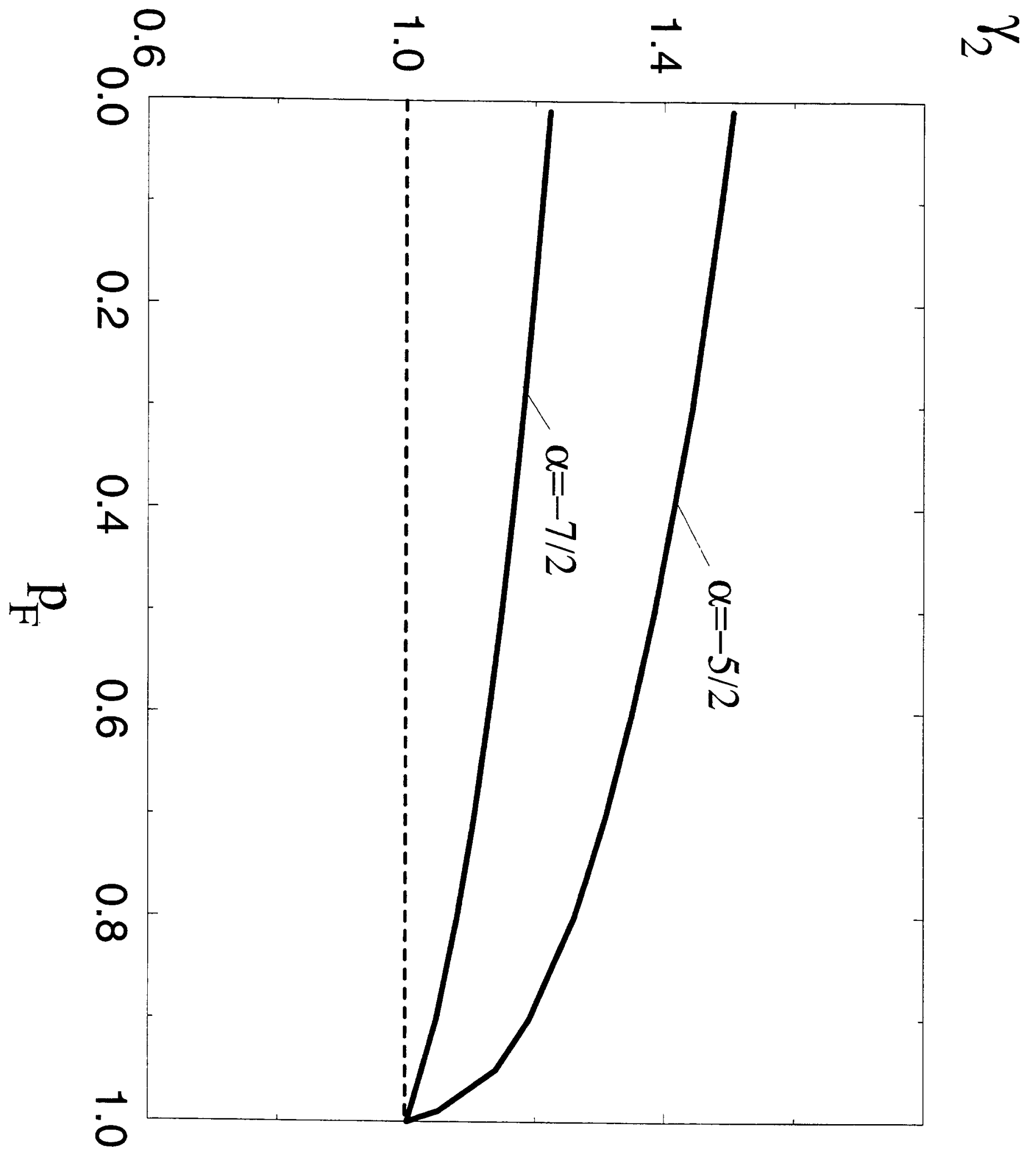

Fig. 9 


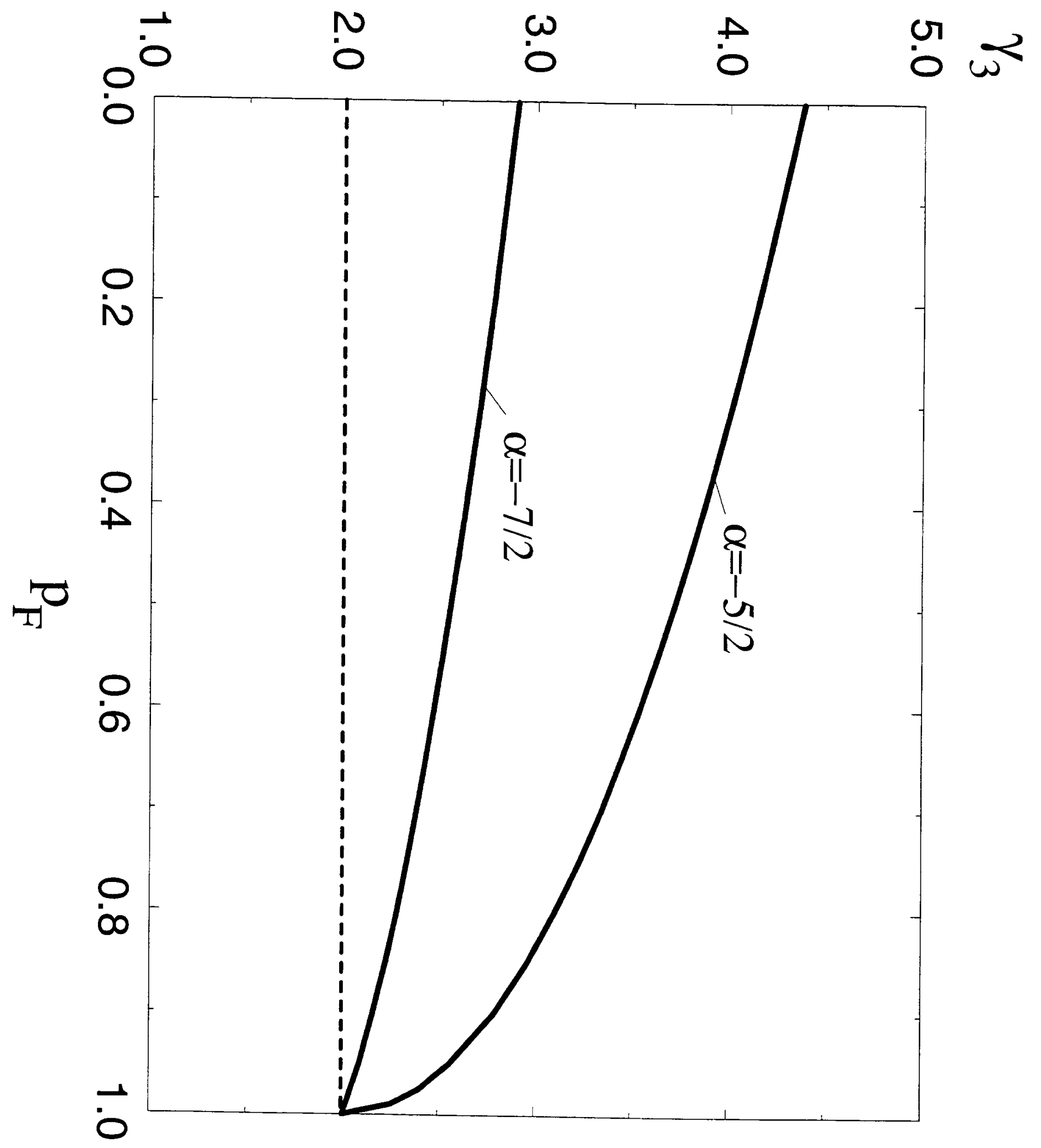

Fig.10 


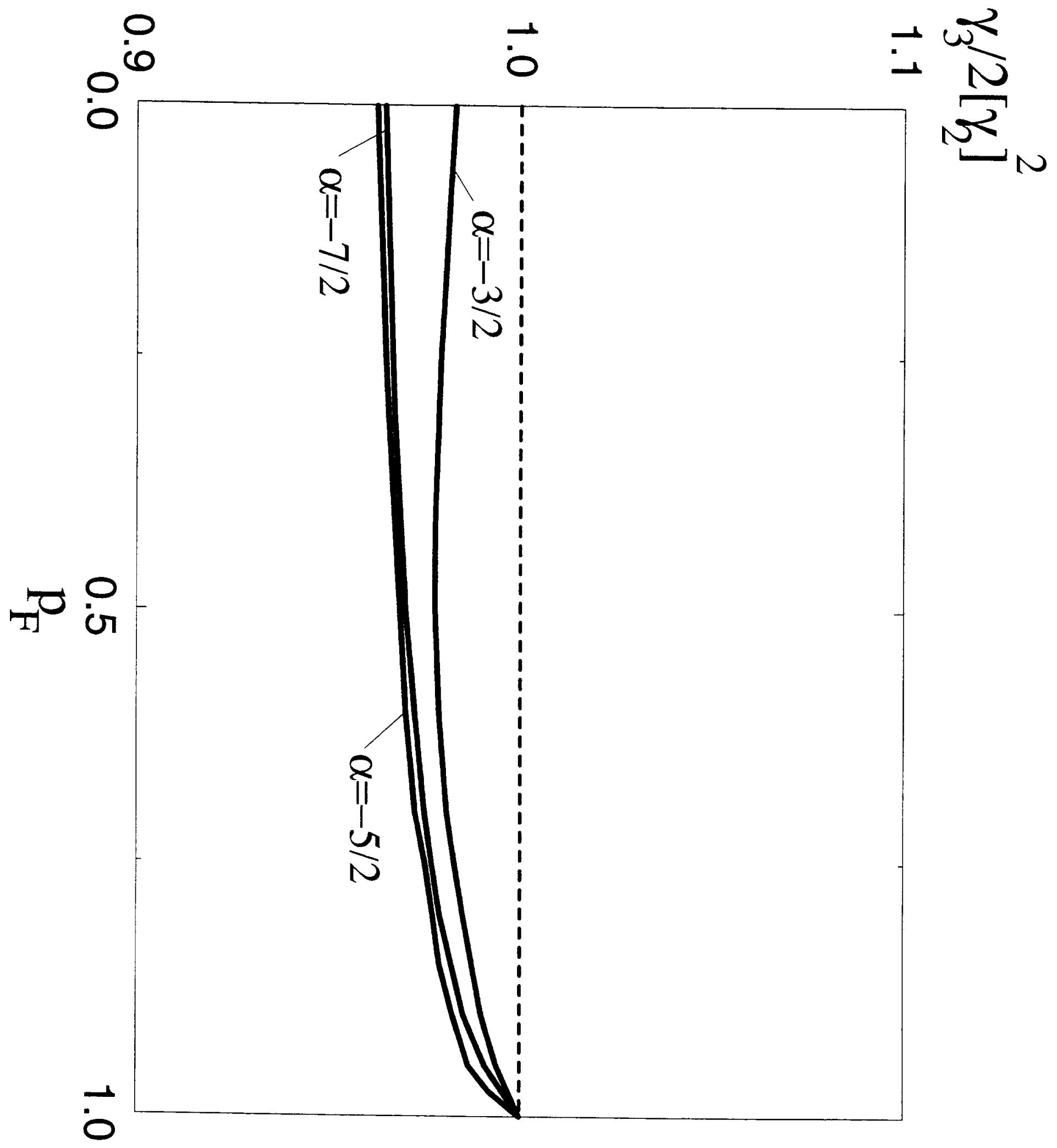

Fig. 11 


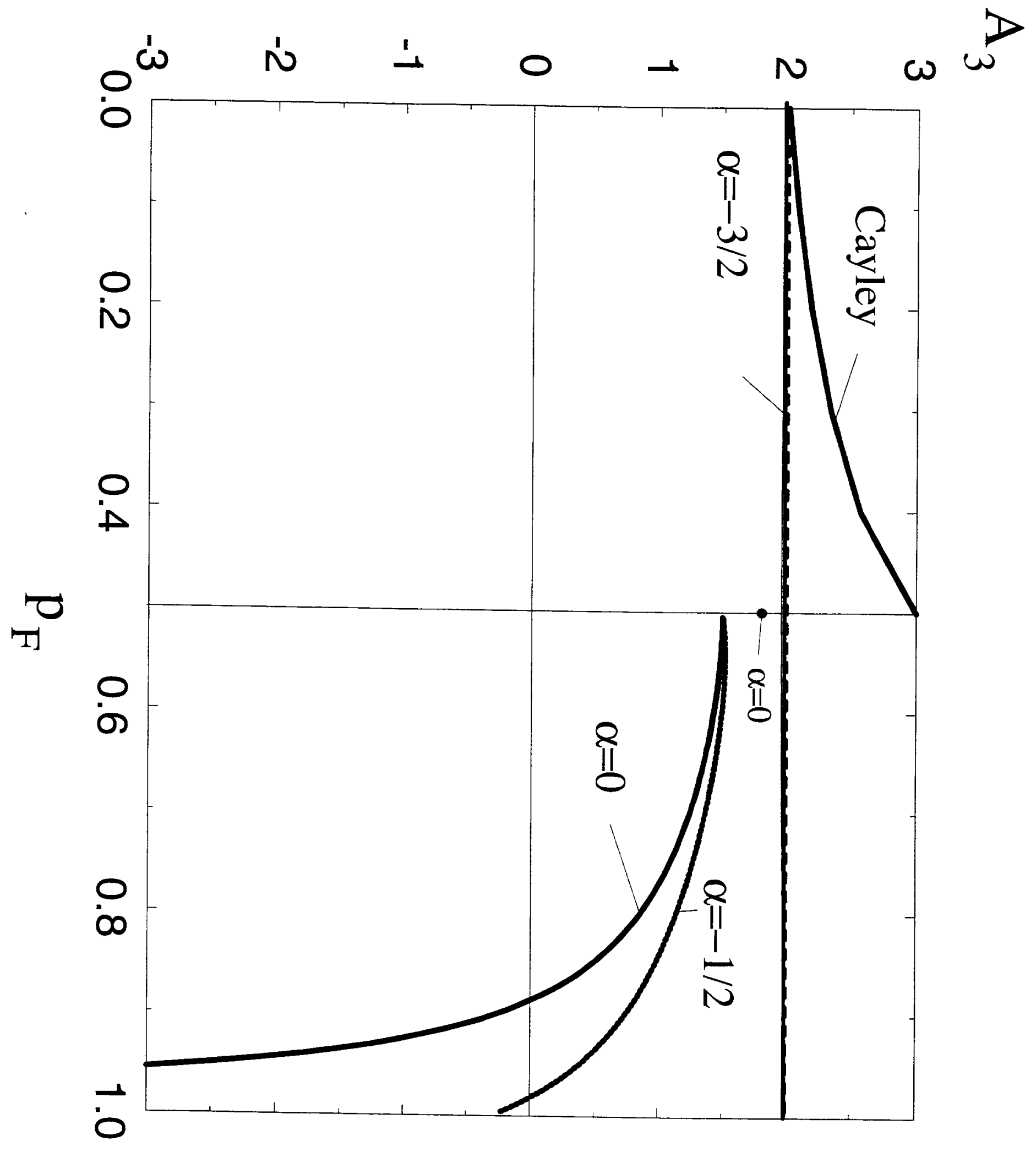

Fig. 12 


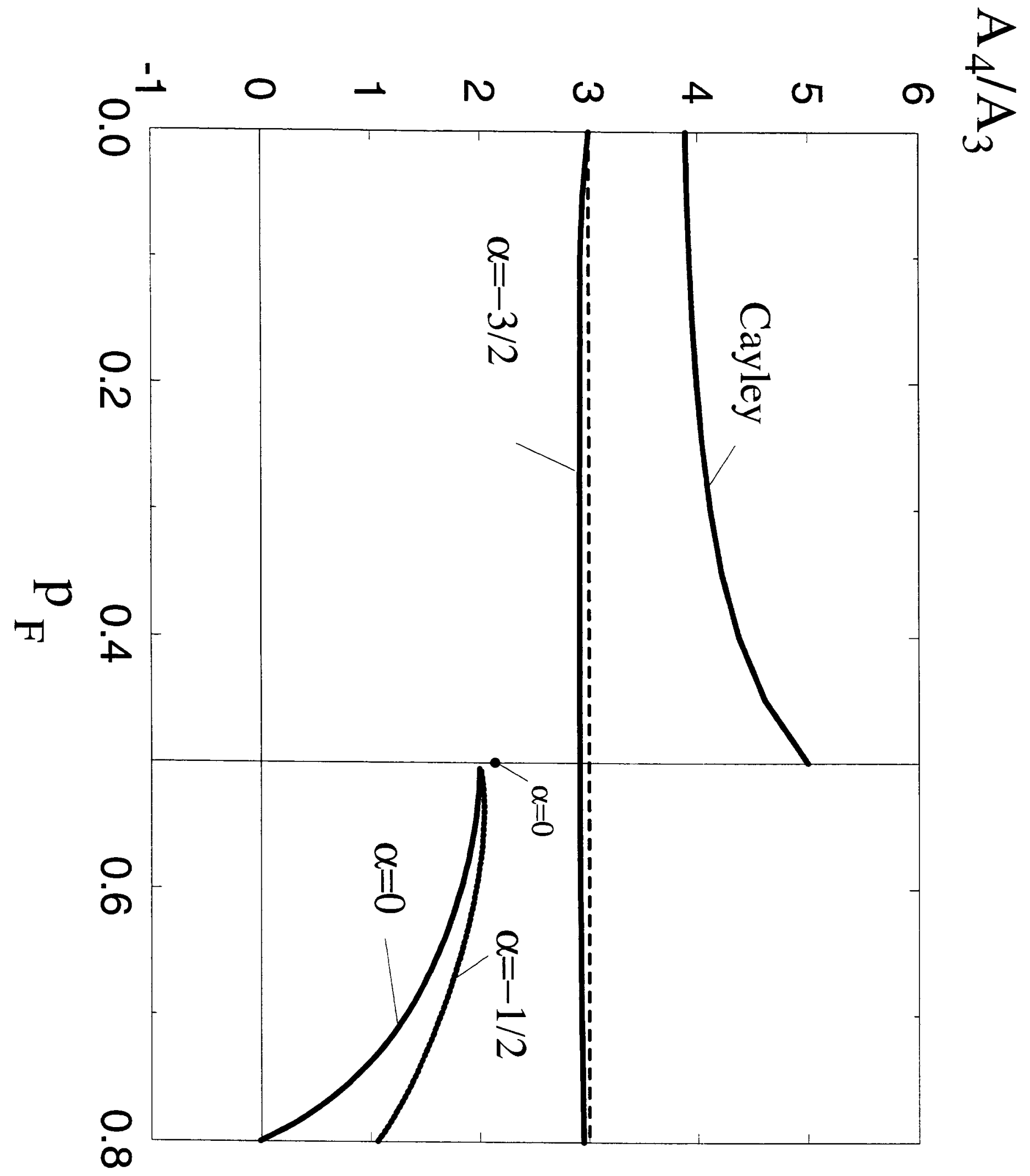

Fig. 13 